\title{
Modelling of a two prey and one predator system with switching effect
}

https://doi.org/10.1515/cmb-2020-0120

Received April 3, 2021; accepted June 18, 2021

Abstract: Prey switching strategy is adopted by a predator when they are provided with more than one prey and predator prefers to consume one prey over others. Though switching may occur due to various reasons such as scarcity of preferable prey or risk in hunting the abundant prey. In this work, we have proposed a prey-predator system with a particular type of switching functional response where a predator feeds on two types of prey but it switches from one prey to another when a particular prey population becomes lower. The ratio of consumption becomes significantly higher in the presence of prey switching for an increasing ratio of prey population which satisfies Murdoch's condition [15]. The analysis reveals that two prey species can coexist as a stable state in absence of predator but a single prey-predator situation cannot be a steady state. Moreover, all the population can coexist only under certain restrictions. We get bistability for a certain range of predation rate for first prey population. Moreover, varying the mortality rate of the predator, an oscillating system can be obtained through Hopf bifurcation. Also, the predation rate for the first prey can turn a steadystate into an oscillating system. Except for Hopf bifurcation, some other local bifurcations also have been studied here. The figures in the numerical simulation have depicted that, if there is a lesser number of one prey present in a system, then with time, switching to the other prey, in fact, increases the predator population significantly.

Keywords: Prey-predator model; Switching effect; Extinction; Bifurcations; Persistence

MSC: 92D25; 92D40; 34C23

\section{Introduction}

For two-prey and one-predator system, the growth rate of a predator population not only depends on the total amount of consumed prey but also on the ratio of the captured prey species. If $x_{1}, x_{2}$ be the densities of two prey which are consumed at rates $h_{1}, h_{2}$ by the predator respectively, then the work of Tinbergen [29] suggests that the "risk index" of the prey is denoted by $r_{i}=\frac{h_{i}}{x_{i}},(i=1,2)$. If the first prey $x_{1}$ is consumed by the predator at a higher rate than the second prey $x_{2}$, then $r_{2}$ is dominated by $r_{1}$. A predator, which is provided with more than one resource population, may try to consume particularly those prey frequently which are abundant at any time and here come the terms 'switching property of predation'. The predator prefers to consume disproportionately according to the adequacy of the prey population in case of prey switching and so, it is a frequency-dependent predation strategy. Here the predator has a choice to select between different prey species. Murdoch (1969), in his work, explained that in the case of prey switching, the relative preference $\frac{r_{1}}{r_{2}}$ maintains a proportionality with relative prey density $\frac{x_{1}}{x_{2}}$ [15]. Prey switching mainly describes a situation when predator prefers a particular prey over others due to their abundance and availability in the environment, i.e., in some cases, there exists a strong preference for those prey which can be easily found and a weak preference for those prey which are available in a smaller quantity. This preference is obtained

Sangeeta Saha: Department of Mathematics, Indian Institute of Engineering Science and Technology, Shibpur, Howrah 711103, India, E-mail: sangeetasaha629@gmail.com

*Corresponding Author: Guruprasad Samanta: Department of Mathematics, Indian Institute of Engineering Science and Technology, Shibpur, Howrah - 711103, India, E-mail: g_p_samanta@yahoo.co.uk, gpsamanta@math.iiests.ac.in 
from the relationship between ratio of prey available in the system and ratio of prey consumed by the predator which is defined as: $\frac{h_{1}}{h_{2}}=c\left(\frac{x_{1}}{x_{2}}\right)$; where $x_{1}, x_{2}$ respectively be the biomass of first and second prey population which are consumed at rates $h_{1}, h_{2}$ by the predator. Here $c$ is the term which represents the preference of the predator for first prey species $\left(x_{1}\right)$, i.e., $c>1$ implies predator's preference for first prey and $c<1$ implies predator's preference for second prey. So, if $c$ starts to increase with the increase of $\frac{x_{1}}{x_{2}}$, it indicates that the predator switches to $x_{1}$ with time. On the other hand, if predator searches for the prey which is present in lesser quantity in the system, then $c$ decreases with an increase of $\frac{x_{1}}{x_{2}}$ and the opposite prey switching occurs. Now, prey switching may occur due to different reasons: in a system, if a predator always consumes the easily available prey, then with time, that prey population decrease and the predator, on that situation, have to look for other prey; secondly, those prey whose abundance is higher can be riskier to hunt.

Switching of prey increases the predator's ability to consume more than one prey instead of sticking up to one particular prey and it increases their growth with a higher rate too. The occurrence of switching behaviour sometimes depends on the environment as well as on the foraging timing. The snail Acanthina in rocky shores of Southern California mainly hunt two types of prey named mussel (Mytilus edulis) and barnacle (Balanus glandula). Oaten \& Murdoch (1975), in their experiment, has concluded that switching occurs for the snails when the prey ratios are varied and also the patches of the abundant prey is provided before experiment [19]. So, prey switching occurs for the higher prey density in this case. On the other hand, in an experiment [17], Guppies have been taken as predator and it has been observed that they hunt for Tubificids and Drosophila on the bottom and at the top of an aquarium respectively. The authors have concluded here that the switching behaviour occurs when the prey density is lower and the predator tries to consume more when the prey is scarce and if both prey species are scarce, then they hunt according to their adequacy. Next, let us consider the case for stoneflies. Elliott (2004), in his experiment, has shown that the mature larvae of Dinocras cephalotes and Perla bipunctata do not show any switching behaviour but the larvae of Isoperla grammatica and Perlodes microcephalus prefer to consume Baetis rhodani over Chironomus $s p$. when the abundance of Baetis is higher in the system [5]. Some literatures reveal that predator can switch the prey population at the time of consumption due to the formation of a search image [9]. It has been observed through experiment that Bombus pensylvanicus exhibits a kind of switching behaviour due to formation of search images [21]. Moreover, in the case when a predator is hunting a particular prey species for a long time, then they become efficient to hunt more of those prey and this leads to the occurrence of prey switching. This is observed in the experiment of Bergelson (1985): the dragonfly Anax junius always go for either mayfly nymphs or tubifex worms according to the prey abundance, i.e., the predator always try to hunt those prey which they have already captured before [3]. Switching of prey population mainly helps a system to become stable and in fact, there are some results show that a system where two prey population coexist becomes stable in the presence of prey switching [1]. One reason for this situation is that predator starts searching for another resource when a particular prey population becomes very low and rare and this can create prey refugia and that prey population can save themselves from the verge of extinction [7].

There are some research works reveal that switching effect make a significant impact on the stability of the prey species [16, 25, 27]. Comins \& Hassell in 1976 and Teramoto et al. in 1979 studied two prey- one predator species where the prey species involve in intra-specific competition $[4,28]$. According to their results, the competing prey species can coexist in the presence of switching effect. Matsuda et al. (1986), in their work, proposed two-trophic and three-trophic systems to analyze the effect of predator switching on stability [11]. Baalen et al. (2001), in their work, have considered that the predator population switch to constant alternative food when the preferred prey is scarce [30]. They have concluded that switching to alternative food does not stabilize the equilibrium but promote persistence. Further Siddon and Witman, in 2000, performed two years duration field caging experiments on the Isles of Shoals in Maine (USA) to analyse behaviorally mediated indirect effects in shallow subtidal food-web [26]. They observed the predation of crab (Cancer borealis) on sea urchins (Strongylocentrotus droebachiensis) in three different habitats named codium fragile algal beds, barrens and mussel beds to analyse the impact of prey switching. They concluded that crabs consume more urchins in the codium and barren habitats than the mussel habitat. In the mussel habitat, crabs start to feed on mussels instead of urchins and here the prey switching occurs. On the other hand, in barren habitat, crabs consume more urchins which indirectly increase the density of Diplosoma spongiforme from Ascidiacea class. 
TANSKY (1978), in their work, have considered a general switching function in such a manner that the 'risk index' of prey increases when its density increases and decreases when the other prey population increases [27]. So, the prey switching occurs according to the prey abundance in a system, i.e., $\frac{\partial r_{1}}{\partial x_{1}}>0, \frac{\partial r_{1}}{\partial x_{2}}<0$ and $\frac{\partial r_{2}}{\partial x_{1}}<0, \frac{\partial r_{2}}{\partial x_{2}}>0$. They have concluded that the switching towards one prey becomes stronger when that prey intensity becomes adequate in the system. There are some literatures consist of general switching functional response which considers that the predator fractionally allocates the searching efforts for each prey $[2,11]$.

The complexity of any model mainly depends on the choice of functional responses. Though Lotka and Volterra first incorporated the prey-predator interaction, later several works have been done by refining the model to observe different dynamical behaviour [12, 14, 22, 23, 24]. In this work, we have shown that the inclusion of some relevant biological arguments has made the model system more realistic. A prey-predator interaction, where the predator's preference over the food is considered, shows more biologically relevant properties as the extinction of predator does not fully depend on the extinction of a particular prey species. So, in this literature prey-predator interaction with switching behaviour of predator is proposed to observe its impact on the dynamics. Here, both the prey are engaged in intra-specific competition and grow with respective logistic growth rate. In the following section, two prey - one predator system is formulated with prey switching, i.e., where the predator chooses the prey population for consumption depending on their abundance in the system. Section 3 shows that the proposed system is biologically well-posed as both prey and predator species are positive and bounded with time. Extinction criterion of the predator is stated in Section 4 and the equilibria with the feasibility criterion are described in Section 5. Local stability analysis and persistence of the system are discussed in Sections 6 and 7 respectively. The conditions for which equilibria change their stability through bifurcations are stated in Section 8 . The global stability criteria are discussed in Section 9 and moreover, the numerical simulation in Section 10 support the analytical findings. The work is ended with a brief conclusion about the findings.

\section{Mathematical Model: Basic Equations}

An ecological system in terms of mathematical equations mainly provides the proper insight into the system and it becomes easier to find the dynamics of the model with time. In this work, a prey-predator interaction is considered with two prey species where the predator population exhibits switching behaviour when a prey quantity becomes smaller. The complex and rich dynamics induced by the switching effect is mainly highlighted in this work.

The proposed system consists of two prey $\left(x_{1}, x_{2}\right)$ and one predator species $(y)$. It is assumed that in absence of predator population, first prey population $\left(x_{1}\right)$ grows according to logistic law with intrinsic growth rate $r_{1}$ and carrying capacity $K_{1}$ and the second prey species $\left(x_{2}\right)$ grows logistically with intrinsic growth rate $r_{2}$ and carrying capacity $K_{2}$. The parameters $\eta_{1}$ and $\eta_{2}$ represent the consumption rate coefficients of the predator on first and second prey species respectively. The predator is assumed to be specialist one as they live depending on only these particular two prey species. In that case, let, $c_{1}$ and $c_{2}$ be the biomass conversion rates for first and second prey species respectively. The parameter $d$ denotes the mortality rate coefficient of the predator population. It is later observed that such switching effect helps to increase the growth of the predator population. First we consider Lotka-Volterra equations with two prey and one predator system in (2.1):

$$
\begin{aligned}
\frac{d x_{1}}{d t} & =r_{1} x_{1}\left(1-\frac{x_{1}}{K_{1}}\right)-\eta_{1} x_{1} y, \\
\frac{d x_{2}}{d t} & =r_{2} x_{2}\left(1-\frac{x_{2}}{K_{2}}\right)-\eta_{2} x_{2} y, \\
\frac{d y}{d t} & =c_{1} \eta_{1} x_{1} y+c_{2} \eta_{2} x_{2} y-d y
\end{aligned}
$$

In system (2.1), the consumption rate of predator for first (or, second) prey depends on that particular prey only. In Holling type functional responses, the consumption rate coefficient is a single parameter $\left(\eta_{1}\right.$ or $\left.\eta_{2}\right)$ 


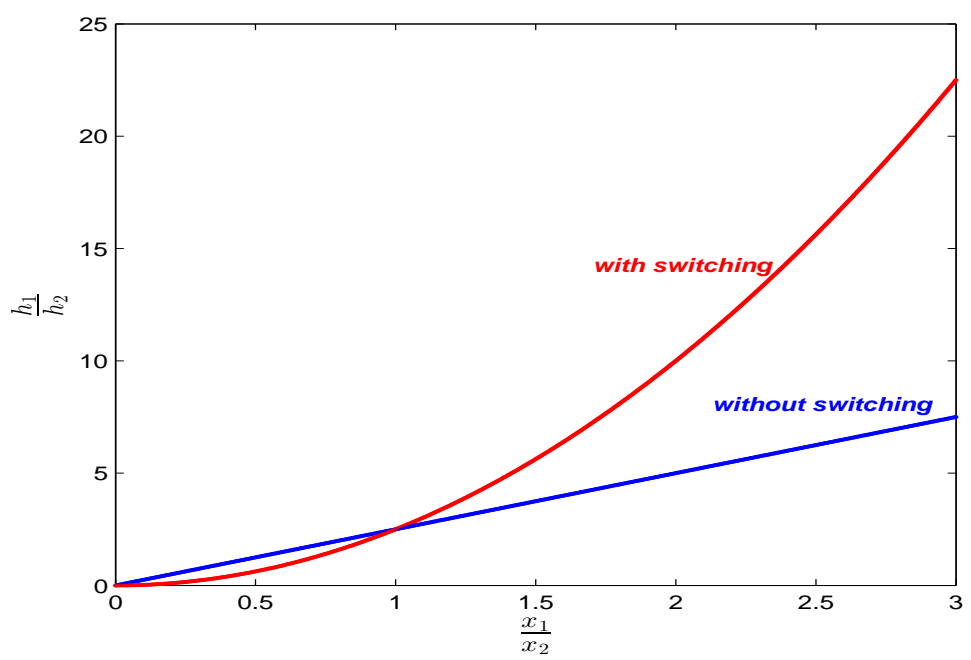

Figure 1: The ratio of amounts of two-prey population consumed by the predator in presence as well as in absence of prey switching. Here $\gamma_{1}=0.5$ and $\gamma_{2}=0.2$.

actually. Now we consider a situation when predator searches for second prey in scarcity of first prey and vice-versa. In this case, let us replace $\eta_{1}$ by $\frac{\gamma_{1} x_{1}}{1+x_{1}+x_{2}}$ and $\eta_{2}$ by $\frac{\gamma_{2} x_{2}}{1+x_{1}+x_{2}}$. When $x_{1}$ is much smaller than $x_{2}$, i.e., $x_{1} \ll x_{2}$, then $1+\frac{1}{x_{1}}+\frac{x_{2}}{x_{1}}$ becomes a large quantity which implies $\eta_{1} \rightarrow 0$ for very small $x_{1}$ (compare to $x_{2}$ ). So, in the scarcity of $x_{1}$, predator switches to $x_{2}$ population for consumption. On the contrary, when $x_{2}$ is much smaller than $x_{1}$, i.e., $x_{2} \ll x_{1}$, then $1+\frac{1}{x_{2}}+\frac{x_{1}}{x_{2}}$ becomes a large quantity which implies $\eta_{2} \rightarrow 0$ for very small $x_{2}$ (compare to $x_{1}$ ). So, when there is a very small amount of second prey population $\left(x_{2}\right)$ exists in the system, the predator switches to first prey population $\left(x_{1}\right)$ for consumption. As a consequence, the predator population switches to that prey which is found to be adequate in the system. This is a kind of density dependence predatory rate which diminishes at low prey density. This may occurs when the prey population becomes rare and the predator switches the predation to another prey species and also the individuals of small prey population find enough hiding places. Considering the switching effect of the predator, system (2.1) becomes:

$$
\begin{aligned}
\frac{d x_{1}}{d t} & =r_{1} x_{1}\left(1-\frac{x_{1}}{K_{1}}\right)-\frac{\gamma_{1} x_{1}^{2} y}{1+x_{1}+x_{2}}, x_{1}(0)>0, \\
\frac{d x_{2}}{d t} & =r_{2} x_{2}\left(1-\frac{x_{2}}{K_{2}}\right)-\frac{\gamma_{2} x_{2}^{2} y}{1+x_{1}+x_{2}}, x_{2}(0)>0, \\
\frac{d y}{d t} & =\frac{c_{1} \gamma_{1} x_{1}^{2} y}{1+x_{1}+x_{2}}+\frac{c_{2} \gamma_{2} x_{2}^{2} y}{1+x_{1}+x_{2}}-d y, \quad y(0)>0,
\end{aligned}
$$

where the model parameters $r_{1}, r_{2}, K_{1}, K_{2}, \gamma_{1}, \gamma_{2}, c_{1}, c_{2}, d$ are positive constants with $0<c_{1}, c_{2}<1$. If we consider $h_{1}$ and $h_{2}$ be the predation rates of the predator for first and second prey species respectively, then the ratio of the predation rates in absence and in presence of switching effect become $\frac{h_{1}}{h_{2}}=u\left(\frac{x_{1}}{x_{2}}\right)$ and $\frac{h_{1}}{h_{2}}=u\left(\frac{x_{1}}{x_{2}}\right)^{2}$ respectively where $u=\frac{\gamma_{1}}{\gamma_{2}}$. Figure 1 shows that the ratio of consumption rate is much higher when switching effect is considered in the system that helps to grow predator population. 


\section{Positivity and Boundedness}

We check positivity and boundedness of the variables of system (2.2) which guarantee the well-posedness.

Theorem 3.1. Solutions of system (2.2) starting in $\mathbb{R}_{+}^{3}$ are positive for $t>0$.

Proof. Right hand sides of systems (2.2) are continuous and locally Lipschitzian on $\mathbb{C}$ implies $\left(x_{1}(t), x_{2}(t), y(t)\right)$ of $(2.2)$ exists and is unique on the interval $[0, \tau)$, where $0<\tau \leq+\infty$ [8]. From the first equation of (2.2), we get

$$
\begin{aligned}
& \frac{d x_{1}}{d t}=r_{1} x_{1}\left(1-\frac{x_{1}}{K_{1}}\right)-\frac{\gamma_{1} x_{1}^{2} y}{1+x_{1}+x_{2}}, \\
& \text { i.e., } x_{1}(t)=x_{1}(0) \exp \left[\int_{0}^{t}\left\{r_{1}\left(1-\frac{x_{1}(s)}{K_{1}}\right)-\frac{\gamma_{1} x_{1}(s) y(s)}{1+x_{1}(s)+x_{2}(s)}\right\} d s\right] \\
& \quad>0 \text {, for } x_{1}(0)>0 .
\end{aligned}
$$

Also, the second equation of (2.2) gives

$$
\begin{aligned}
\frac{d x_{2}}{d t}=r_{2} x_{2}\left(1-\frac{x_{2}}{K_{2}}\right)-\frac{\gamma_{2} x_{2}^{2} y}{1+x_{1}+x_{2}}, \\
\Rightarrow x_{2}(t)=x_{2}(0) \exp \left[\int_{0}^{t}\left\{r_{2}\left(1-\frac{x_{2}(s)}{K_{2}}\right)-\frac{\gamma_{2} x_{2}(s) y(s)}{1+x_{1}(s)+x_{2}(s)}\right\} d s\right] \\
>0, \text { for } x_{2}(0)>0 .
\end{aligned}
$$

The last equation of system (2.2) gives

$$
y(t)=y_{0} \exp \left[\int_{0}^{t}\left\{\frac{c_{1} \gamma_{1} x_{1}(s)^{2}}{1+x_{1}(s)+x_{2}(s)}+\frac{c_{2} \gamma_{2} x_{2}(s)^{2}}{1+x_{1}(s)+x_{2}(s)}-d\right\} d s\right]>0 \text {, for } y(0)>0 .
$$

Theorem 3.2. Solutions of system (2.2) starting in $\mathbb{R}_{+}^{3}$ remain uniformly bounded for $t>0$.

Proof. From the first equation of (2.2):

$$
\begin{aligned}
\frac{d x_{1}}{d t} & =r_{1} x_{1}\left(1-\frac{x_{1}}{K_{1}}\right)-\frac{\gamma_{1} x_{1}^{2} y}{1+x_{1}+x_{2}} \\
& \leq r_{1} x_{1}\left(1-\frac{x_{1}}{K_{1}}\right) \\
\Rightarrow & \lim \sup _{t \rightarrow \infty} x_{1}(t) \leq K_{1} .
\end{aligned}
$$

Further from the second equation,

$$
\begin{aligned}
\frac{d x_{2}}{d t} & =r_{2} x_{2}\left(1-\frac{x_{2}}{K_{2}}\right)-\frac{\gamma_{2} x_{2}^{2} y}{1+x_{1}+x_{2}} \\
& \leq r_{2} x_{2}\left(1-\frac{x_{2}}{K_{2}}\right) \\
\Rightarrow & \limsup _{t \rightarrow \infty} x_{2}(t) \leq K_{2} .
\end{aligned}
$$


Let $W=c_{1} x_{1}+c_{2} x_{2}+y$

So, for large time $t: \frac{d W}{d t}=c_{1} r_{1} x_{1}\left(1-\frac{x_{1}}{K_{1}}\right)+c_{2} r_{2} x_{2}\left(1-\frac{x_{2}}{K_{2}}\right)-d y$

$$
\begin{aligned}
& \leq c_{1} r_{1} x_{1}+c_{2} r_{2} x_{2}-d y \\
& \leq c_{1}\left(1+r_{1}\right) K_{1}+c_{2}\left(1+r_{2}\right) K_{2}-\kappa W, \text { where } \kappa=\min \{1, d\} \\
\therefore \frac{d W}{d t}+\kappa W & \leq c_{1}\left(1+r_{1}\right) K_{1}+c_{2}\left(1+r_{2}\right) K_{2} \\
\Rightarrow W(t) & \leq \frac{\left\{c_{1}\left(1+r_{1}\right) K_{1}+c_{2}\left(1+r_{2}\right) K_{2}\right\}}{\kappa}\left(1-e^{-\tau t}\right)+W\left(x_{1}(0), x_{2}(0), y(0)\right) e^{-\kappa t} .
\end{aligned}
$$

As $t \rightarrow \infty, 0<W(t) \leq \frac{\left\{c_{1}\left(1+r_{1}\right) K_{1}+c_{2}\left(1+r_{2}\right) K_{2}\right\}}{\kappa}$.

Therefore, all solutions of system (2.2) enter into the region:

$$
\Omega=\left\{\left(x_{1}, x_{2}, y\right): 0<x_{1}(t) \leq K_{1} ; 0<x_{2}(t) \leq K_{2} ; 0<W(t) \leq \frac{1}{\kappa}\left\{c_{1}\left(1+r_{1}\right) K_{1}+c_{2}\left(1+r_{2}\right) K_{2}\right\}\right\} .
$$

\section{Extinction Scenarios}

Following theorem provides the criterion for which predator population goes extinct from the system in long run.

Theorem 4.1. If $c_{1} \gamma_{1} K_{1}^{2}+c_{2} \gamma_{2} K_{2}^{2}<d$, then $\lim _{t \rightarrow \infty} y=0$.

Proof. For large time $t$ :

$$
\begin{aligned}
\frac{d y}{d t} & =\frac{c_{1} \gamma_{1} x_{1}^{2} y}{1+x_{1}+x_{2}}+\frac{c_{2} \gamma_{2} x_{2}^{2} y}{1+x_{1}+x_{2}}-d y \\
& \leq c_{1} \gamma_{1} x_{1}^{2} y+c_{2} \gamma_{2} x_{2}^{2} y-d y \\
& \leq\left(c_{1} \gamma_{1} K_{1}^{2}+c_{2} \gamma_{2} K_{2}^{2}-d\right) y \\
& =-\mu y \quad\left[\text { where, } \mu=\left(d-c_{1} \gamma_{1} K_{1}^{2}-c_{2} \gamma_{2} K_{2}^{2}\right)>0\right]
\end{aligned}
$$

Hence, $\lim _{t \rightarrow \infty} y=0$.

Remark. If the overall growth rate of predator fails to exceeds its mortality rate, then with time, the predator population goes extinct from the system.

\section{Equilibrium Points}

The proposed system (2.2) has the following equilibrium points:

1. Trivial Equilibrium Point: $E_{0}(0,0,0)$.

2. Axial Equilibrium Points: (i) $E_{1}\left(K_{1}, 0,0\right)$ and (ii) $E_{2}\left(0, K_{2}, 0\right)$.

3. Planar Equilibrium Points: (i) $E_{3}\left(K_{1}, K_{2}, 0\right)$, (ii) $E_{4}\left(\overline{x_{1}}, 0, \bar{y}\right)$ where $\overline{x_{1}}=\frac{d+\sqrt{d^{2}+4 d c_{1} \gamma_{1}}}{2 c_{1} \gamma_{1}}$ and $\bar{y}=\frac{r_{1}}{\gamma_{1}}\left(\frac{1+\overline{x_{1}}}{\overline{x_{1}}}\right)\left(1-\frac{\overline{x_{1}}}{K_{1}}\right)$; (iii) $E_{5}\left(0, \underline{x_{2}}, \underline{y}\right)$ where $\underline{x_{2}}=\frac{d+\sqrt{d^{2}+4 d c_{2} \gamma_{2}}}{2 c_{2} \gamma_{2}}$ and $\underline{y}=$ $\frac{r_{2}}{\gamma_{2}}\left(\frac{1+\underline{x_{2}}}{\underline{x_{2}}}\right)\left(1-\frac{x_{2}}{\bar{K}_{2}}\right)$ 
4. Interior Equilibrium Point $E^{\star}\left(x_{1}^{\star}, x_{2}^{\star}, y^{\star}\right)$ where $y^{\star}=\frac{r_{1}}{\gamma_{1} x_{1}^{\star}}\left(1+x_{1}^{\star}+x_{2}^{\star}\right)\left(1-\frac{x_{1}^{\star}}{K_{1}}\right)$ and $x_{2}^{\star}=$ $\frac{r_{2} \gamma_{1} K_{1} K_{2} x_{1}^{\star}}{\left\{r_{1} \gamma_{2} K_{1} K_{2}+P x_{1}^{\star}\right\}}$ with $P=r_{2} \gamma_{1} K_{1}-r_{1} \gamma_{2} K_{2}$ and $x_{1}^{\star}$ is the positive root of the equation:

$$
G\left(x_{1}\right) \equiv C_{1} x_{1}^{4}+C_{2} x_{1}^{3}+C_{3} x_{1}^{2}+C_{4} x_{1}+C_{5}=0,
$$

where, $C_{1}=c_{1} \gamma_{1} P^{2}>0$,

$$
\begin{aligned}
& C_{2}=P\left(2 c_{1} \gamma_{1} r_{1} \gamma_{2} K_{1} K_{2}-P d\right), \\
& C_{3}=K_{1}^{2} K_{2}^{2}\left(c_{1} \gamma_{2} r_{1}^{2}+c_{2} \gamma_{1} r_{2}^{2}\right) \gamma_{1} \gamma_{2}-d P r_{1} \gamma_{2} K_{1} K_{2}-d P^{2}-d P\left(r_{1} \gamma_{2}+r_{2} \gamma_{1}\right) K_{1} K_{2}, \\
& C_{4}=-d\left[r_{1} \gamma_{2} K_{1} K_{2}\left\{P+\left(r_{1} \gamma_{2}+r_{2} \gamma_{1}\right) K_{1} K_{2}\right\}+P r_{1} \gamma_{2} K_{1} K_{2}\right], \\
& C_{5}=-d\left(r_{1} \gamma_{2} K_{1} K_{2}\right)^{2}<0 .
\end{aligned}
$$

\section{Local Stability Analysis}

Local stability conditions of the equilibrium points can be determined by the eigenvalues of the corresponding Jacobian matrices. Now, the Jacobian matrix of system (2.2) is given by

$$
J=\left(\begin{array}{lll}
a_{11} & a_{12} & a_{13} \\
a_{21} & a_{22} & a_{23} \\
a_{31} & a_{32} & a_{33}
\end{array}\right),
$$

where $a_{11}=r_{1}\left(1-\frac{2 x_{1}}{K_{1}}\right)-\frac{\gamma_{1} y\left\{2 x_{1}\left(1+x_{2}\right)+x_{1}^{2}\right\}}{\left(1+x_{1}+x_{2}\right)^{2}} ; a_{12}=\frac{\gamma_{1} x_{1}^{2} y}{\left(1+x_{1}+x_{2}\right)^{2}} ; a_{13}=-\frac{\gamma_{1} x_{1}^{2}}{1+x_{1}+x_{2}}$;

$a_{21}=\frac{\gamma_{2} x_{2}^{2} y}{\left(1+x_{1}+x_{2}\right)^{2}} ; a_{22}=r_{2}\left(1-\frac{2 x_{2}}{K_{2}}\right)-\frac{\gamma_{2} y\left\{2 x_{2}\left(1+x_{1}\right)+x_{2}^{2}\right\}}{\left(1+x_{1}+x_{2}\right)^{2}} ; a_{23}=-\frac{\gamma_{2} x_{2}^{2}}{1+x_{1}+x_{2}} ;$

$a_{31}=\frac{c_{1} \gamma_{1} y\left\{2 x_{1}\left(1+x_{2}\right)+x_{1}^{2}\right\}}{\left(1+x_{1}+x_{2}\right)^{2}}-\frac{c_{2} \gamma_{2} x_{2}^{2} y}{\left(1+x_{1}+x_{2}\right)^{2}} ; a_{32}=\frac{c_{2} \gamma_{2} y\left\{2 x_{2}\left(1+x_{1}\right)+x_{2}^{2}\right\}}{\left(1+x_{1}+x_{2}\right)^{2}}-\frac{c_{1} \gamma_{1} x_{1}^{2} y}{\left(1+x_{1}+x_{2}\right)^{2}} ;$

$a_{33}=\frac{c_{1} \gamma_{1} x_{1}^{2}}{1+x_{1}+x_{2}}+\frac{c_{2} \gamma_{2} x_{2}^{2}}{1+x_{1}+x_{2}}-d$.

For $E_{0}=(0,0,0):\left.J\right|_{E_{0}}=\left(\begin{array}{ccc}r_{1} & 0 & 0 \\ 0 & r_{2} & 0 \\ 0 & 0 & -d\end{array}\right)$.

So, $\lambda_{1}=r_{1}>0, \lambda_{2}=r_{2}>0$ and $\lambda_{3}=-d<0$.

Theorem 6.1. $E_{0}$ is always a saddle point.

For $E_{1}=\left(K_{1}, 0,0\right):\left.J\right|_{E_{1}}=\left(\begin{array}{ccc}-r_{1} & 0 & -\frac{\gamma_{1} K_{1}^{2}}{1+K_{1}} \\ 0 & r_{2} & 0 \\ 0 & 0 & \frac{c_{1} \gamma_{1} K_{1}^{2}}{1+K_{1}}-d\end{array}\right)$.

So, $\lambda_{1}=-r_{1}<0, \lambda_{2}=r_{2}>0$ and $\lambda_{3}=\frac{c_{1} \gamma_{1} K_{1}^{2}}{1+K_{1}}-d$. So, we have the following theorem:

Theorem 6.2. $E_{1}$ is always a saddle point.

For $E_{2}=\left(0, K_{2}, 0\right):\left.J\right|_{E_{2}}=\left(\begin{array}{ccc}r_{1} & 0 & 0 \\ 0 & -r_{2} & -\frac{\gamma_{2} K_{2}^{2}}{1+K_{2}} \\ 0 & 0 & \frac{c_{2} \gamma_{2} K_{2}^{2}}{1+K_{2}}-d\end{array}\right)$.

So, $\lambda_{1}=r_{1}>0, \lambda_{2}=-r_{2}<0$ and $\lambda_{3}=\frac{c_{2} \gamma_{2} K_{2}^{2}}{1+K_{2}}-d$. So, we have the following theorem: 
Theorem 6.3. $E_{2}$ is always a saddle point.

For $E_{3}=\left(K_{1}, K_{2}, 0\right):\left.J\right|_{E_{3}}=\left(\begin{array}{ccc}-r_{1} & 0 & -\frac{\gamma_{1} K_{1}^{2}}{1+K_{1}+K_{2}} \\ 0 & -r_{2} & -\frac{\gamma_{2} K_{2}^{2}}{1+K_{1}+K_{2}} \\ 0 & 0 & \frac{c_{1} \gamma_{1} K_{1}^{2}}{1+K_{1}+K_{2}}+\frac{c_{2} \gamma_{2} 2_{2}^{2}}{1+K_{1}+K_{2}}-d\end{array}\right)$.

So, $\lambda_{1}=-r_{1}<0, \lambda_{2}=-r_{2}<0$ and $\lambda_{3}=\frac{c_{1} \gamma_{1} K_{1}^{2}}{1+K_{1}+K_{2}}+\frac{c_{2} \gamma_{2} K_{2}^{2}}{1+K_{1}+K_{2}}-d$. So, we have the following theorem:

Theorem 6.4. $E_{3}$ is a stable equilibrium point when $c_{1} \gamma_{1} K_{1}^{2}+c_{2} \gamma_{2} K_{2}^{2}<d\left(1+K_{1}+K_{2}\right)$ and is a saddle point when $c_{1} \gamma_{1} K_{1}^{2}+c_{2} \gamma_{2} K_{2}^{2}>d\left(1+K_{1}+K_{2}\right)$.

Now, for $E_{4}=\left(\overline{x_{1}}, 0, \bar{y}\right):\left.J\right|_{E_{4}}=\left(\begin{array}{ccc}a_{11} & a_{12} & a_{13} \\ 0 & a_{22} & 0 \\ a_{31} & a_{32} & 0\end{array}\right)$,

where $a_{11}=-\left[\frac{r_{1} \overline{x_{1}}}{K_{1}}+\frac{\gamma_{1} \overline{x_{1} y}}{\left(1+\overline{x_{1}}\right)^{2}}\right] ; \quad a_{12}=\frac{\gamma_{1}{\overline{x_{1}}}^{2} \bar{y}}{\left(1+\overline{x_{1}}\right)^{2}} ; a_{13}=-\frac{\gamma_{1}{\overline{x_{1}}}^{2}}{1+\overline{x_{1}}} ; a_{22}=r_{2} ; a_{31}=$ $\frac{c_{1} \gamma_{1} \bar{y}\left\{2 \overline{x_{1}}+{\overline{x_{1}}}^{2}\right\}}{\left(1+\overline{x_{1}}\right)^{2}} ; a_{32}=-\frac{c_{1} \gamma_{1}{\overline{x_{1}}}^{2} \bar{y}}{\left(1+\overline{x_{1}}\right)^{2}}$. So, one eigenvalue is $\lambda_{1}=a_{22}=r_{2}>0$ and other two eigenvalues are roots of the equation: $\lambda^{2}-a_{11} \lambda-a_{13} a_{31}=0$. So, two eigenvalues have negative real parts but one eigenvalue is always positive.

Theorem 6.5. $E_{4}$ is always a saddle point.

Now, for $E_{5}=\left(0, \underline{x_{2}}, \underline{y}\right):\left.J\right|_{E_{5}}=\left(\begin{array}{ccc}a_{11} & 0 & 0 \\ a_{21} & a_{22} & a_{23} \\ a_{31} & a_{32} & 0\end{array}\right)$,

where $a_{11}=r_{1} ; a_{21}=\frac{\gamma_{2}{\underline{x_{2}}}^{2} \underline{y}}{\left(1+\underline{x_{2}}\right)^{2}} ; a_{22}=-\left[\frac{r_{2} \underline{x_{2}}}{K_{2}}+\frac{\gamma_{2} \underline{x_{2}} \underline{y}}{\left(1+\underline{x_{2}}\right)^{2}}\right] ; a_{23}=-\frac{\gamma_{2}{\underline{x_{2}}}^{2}}{1+\underline{x_{2}}}$;

$a_{31}=-\frac{c_{2} \gamma_{2} \underline{x_{2}}{ }^{2} y}{\left(1+\underline{x_{2}}\right)^{2}} ; a_{32}=\frac{c_{2} \gamma_{2} \underline{y}\left\{2 \underline{x_{2}}+{\underline{x_{2}}}^{2}\right\}}{\left(1+{\underline{x_{2}}}^{2}\right)^{2}}$. So, one eigenvalue is $\lambda_{1}=a_{11}=r_{1}>0$ and other two eigenvalues are roots of the equation: $\lambda^{2}-a_{22} \lambda-a_{23} a_{32}=0$. So, two eigenvalues have negative real parts but one eigenvalue is always positive.

Theorem 6.6. $E_{5}$ is always a saddle point.

Now, for $E^{\star}\left(x_{1}^{\star}, x_{2}^{\star}, y^{\star}\right):\left.J\right|_{E^{\star}}=\left(\begin{array}{ccc}a_{11} & a_{12} & a_{13} \\ a_{21} & a_{22} & a_{23} \\ a_{31} & a_{32} & 0\end{array}\right)$,

where, $a_{11}=-\left[\frac{r_{1} x_{1}^{\star}}{K_{1}}+\frac{\gamma_{1} x_{1}^{\star} y\left(1+x_{2}^{\star}\right)}{\left(1+x_{1}^{\star}+x_{2}^{\star}\right)^{2}}\right] ; a_{12}=\frac{\gamma_{1} x_{1}^{\star} y}{\left(1+x_{1}^{\star}+x_{2}^{\star}\right)^{2}} ; a_{13}=-\frac{\gamma_{1} x_{1}^{\star 2}}{1+x_{1}^{\star}+x_{2}^{\star}}$;

$a_{21}=\frac{\gamma_{2} x_{2}^{\star 2} y}{\left(1+x_{1}^{\star}+x_{2}^{\star}\right)^{2}} ; a_{22}=-\left[\frac{r_{2} x_{2}^{\star}}{K_{2}}+\frac{\gamma_{2} x_{2}^{\star} y^{\star}\left(1+x_{1}^{\star}\right)}{\left(1+x_{1}^{\star}+x_{2}^{\star}\right)^{2}}\right] ; a_{23}=-\frac{\gamma_{2} x_{2}^{\star 2}}{1+x_{1}^{\star}+x_{2}^{\star}} ;$

$a_{31}=\frac{c_{1} \gamma_{1} y^{\star}\left\{2 x_{1}^{\star}\left(1+x_{2}^{\star}\right)+x_{1}^{\star 2}\right\}}{\left(1+x_{1}^{\star}+x_{2}^{\star}\right)^{2}}-\frac{c_{2} \gamma_{2} x_{2}^{\star 2} y^{\star}}{\left(1+x_{1}^{\star}+x_{2}^{\star}\right)^{2}} ; a_{32}=\frac{c_{2} \gamma_{2} y^{\star}\left\{2 x_{2}^{\star}\left(1+x_{1}^{\star}\right)+x_{2}^{\star 2}\right\}}{\left(1+x_{1}^{\star}+x_{2}^{\star}\right)^{2}}-\frac{c_{1} \gamma_{1} x_{1}^{\star 2} y^{\star}}{\left(1+x_{1}^{\star}+x_{2}^{\star}\right)^{2}}$.

Characteristic equation for $E^{\star}\left(x_{1}^{\star}, x_{2}^{\star}, y^{\star}\right)$ is

$\lambda^{3}+P_{1} \lambda^{2}+P_{2} \lambda+P_{3}=0$,

where $P_{1}=-\left(a_{11}+a_{22}\right)>0, P_{2}=a_{11} a_{22}-a_{12} a_{21}-a_{13} a_{31}-a_{23} a_{32}$ and $P_{3}=a_{32}\left(a_{11} a_{23}-a_{13} a_{21}\right)+$ $a_{31}\left(a_{13} a_{22}-a_{12} a_{23}\right)$. Consider, $\Delta=P_{1} P_{2}-P_{3}$, and so by Routh-Hurwitz criterion all the roots of equation (6.1) have negative real parts if $P_{3}>0$ and $\Delta>0$. Hence, we have the following theorem: 
Theorem 6.7. Interior equilibrium point $E^{\star}\left(x_{1}^{\star}, x_{2}^{\star}, y^{\star}\right)$ is locally asymptotically stable (LAS) if $P_{3}>0$ along with $P_{1} P_{2}>P_{3}$.

\section{Persistence}

In the ecological context, persistence means the long term survival of all species which exist initially.

Theorem 7.1. System (2.2) is persistent if $c_{1} \gamma_{1} K_{1}^{2}+c_{2} \gamma_{2} K_{2}^{2}>d\left(1+K_{1}+K_{2}\right)$ holds good.

Proof. Let the average Lyapunov function be $V\left(x_{1}, x_{2}, y\right)=x_{1}^{\theta_{1}} x_{2}^{\theta_{2}} y^{\theta_{3}}$ where $\theta_{i}$ for $i=1,2,3$ are positive. In the interior of $\mathbb{R}_{+}^{3}$, we have

$$
\begin{aligned}
\frac{\dot{V}}{V}=\phi\left(x_{1}, x_{2}, y\right) & =\theta_{1}\left[r_{1}\left(1-\frac{x_{1}}{K_{1}}\right)-\frac{\gamma_{1} x_{1} y}{1+x_{1}+x_{2}}\right] \\
& +\theta_{2}\left[r_{2}\left(1-\frac{x_{2}}{K_{2}}\right)-\frac{\gamma_{2} x_{2} y}{1+x_{1}+x_{2}}\right]+\theta_{3}\left[\frac{c_{1} \gamma_{1} x_{1}^{2}}{1+x_{1}+x_{2}}+\frac{c_{2} \gamma_{2} x_{2}^{2}}{1+x_{1}+x_{2}}-d\right]
\end{aligned}
$$

If the system is persistent, then $\phi\left(x_{1}, x_{2}, y\right)>0$ for all boundary equilibria of the system. The values of $\phi\left(x_{1}, x_{2}, y\right)$ at the boundary equilibria $E_{0}, E_{1}, E_{2}, E_{3}, E_{4}$ and $E_{5}$ are as follows:

$E_{0}: \phi(0,0,0)=r_{1} \theta_{1}+r_{2} \theta_{2}-d \theta_{3}$

$E_{1}: \phi\left(K_{1}, 0,0\right)=\theta_{2} r_{2}+\theta_{3}\left(\frac{c_{1} \gamma_{1} K_{1}^{2}}{1+K_{1}}-d\right)$;

$E_{2}: \phi\left(0, K_{2}, 0\right)=r_{1} \theta_{1}+\theta_{3}\left(\frac{c_{2} \gamma_{2} K_{2}^{2}}{1+K_{2}}-d\right)$;

$E_{3}: \phi\left(K_{1}, K_{2}, 0\right)=\theta_{3}\left(\frac{c_{1} \gamma_{1} K_{1}^{2}}{1+K_{1}+K_{2}}+\frac{c_{2} \gamma_{2} K_{2}^{2}}{1+K_{1}+K_{2}}-d\right)$;

$E_{4}: \phi\left(\overline{x_{1}}, 0, \bar{y}\right)=\theta_{2} r_{2}$

$E_{5}: \phi\left(0, \underline{x_{2}}, \underline{y}\right)=\theta_{1} r_{1}$.

So, if $c_{1} \gamma_{1} K_{1}^{2}+c_{2} \gamma_{2} K_{2}^{2}>d\left(1+K_{1}+K_{2}\right)$ holds good, then $\phi\left(x_{1}, x_{2}, y\right)$ is positive at the boundary equilibria $E_{0}, E_{1}, E_{2}, E_{3}, E_{4}$ and $E_{5}$ for some positive $\theta_{i}, i=1,2$, 3. Hence system (2.2) is persistent [6,13] if $c_{1} \gamma_{1} K_{1}^{2}+$ $c_{2} \gamma_{2} K_{2}^{2}>d\left(1+K_{1}+K_{2}\right)$ is satisfied.

Remark: This condition guarantees the instability of the boundary equilibria of system (2.2).

\section{Bifurcation Analysis}

Change of stability around the equilibria have been discussed in this section. We have used Sotomayor's Theorem [20] and the Hopf Bifurcation Theorem [18] to observe the local bifurcation analysis of system (2.2). In order to apply Sotomayor's Theorem, the Jacobian matrix at the bifurcating equilibrium point has to contain one zero eigenvalue.

Let $V=\left(v_{1}, v_{2}, v_{3}\right)^{T}$ and $W=\left(w_{1}, w_{2}, w_{3}\right)^{T}$ be the eigenvectors of $\left.J\right|_{(\text {eq. point })}$ and $\left.J\right|_{(\text {eq. point })} ^{T}$ respectively corresponding to zero eigenvalue at the equilibrium point.

Let $f=\left(f_{1}, f_{2}, f_{3}\right)^{T}$, where

$f_{1}=r_{1} x_{1}\left(1-\frac{x_{1}}{K_{1}}\right)-\frac{\gamma_{1} x_{1}^{2} y}{1+x_{1}+x_{2}}, f_{2}=r_{2} x_{2}\left(1-\frac{x_{2}}{K_{2}}\right)-\frac{\gamma_{2} x_{2}^{2} y}{1+x_{1}+x_{2}}$,

$f_{3}=\frac{c_{1} \gamma_{1} x_{1}^{2} y}{1+x_{1}+x_{2}}+\frac{c_{2} \gamma_{2} x_{2}^{2} y}{1+x_{1}+x_{2}}-d y$.

Theorem 8.1. System (2.2) undergoes a transcritical bifurcation with respect to the bifurcation parameter $d$ around $E_{3}\left(K_{1}, K_{2}, 0\right)$ if $c_{1} \gamma_{1} K_{1}^{2}+c_{2} \gamma_{2} K_{2}^{2}=d\left(1+K_{1}+K_{2}\right)$. 
Proof.

$\left.J\right|_{E_{3}}=\left(\begin{array}{ccc}-r_{1} & 0 & -\frac{\gamma_{1} K_{1}^{2}}{1+K_{1}+K_{2}} \\ 0 & -r_{2} & -\frac{\gamma_{2} K_{2}^{2}}{1+K_{1}+K_{2}} \\ 0 & 0 & \frac{c_{1} \gamma_{1} K_{1}^{2}}{1+K_{1}+K_{2}}+\frac{c_{2} \gamma_{2} K_{2}^{2}}{1+K_{1}+K_{2}}-d\end{array}\right)$

Let, $d_{[T C]}$ be the value of $d$ such that $\left.J\right|_{E_{3}}$ has a simple zero eigenvalue at $d=d_{[T C]}$.

So, at $d=d_{[T C]}$ :

$\left.J\right|_{E_{3}}=\left(\begin{array}{ccc}-r_{1} & 0 & -\frac{\gamma_{1} K_{1}^{2}}{1+K_{1}+K_{2}} \\ 0 & -r_{2} & -\frac{\gamma_{2} K_{2}^{2}}{1+K_{1}+K_{2}} \\ 0 & 0 & 0\end{array}\right)$.

Here, $\lambda_{1}=-r_{1}<0, \lambda_{2}=-r_{2}<0$ and $\lambda_{3}=0$.

After some calculations: $V=\left(r_{2} \gamma_{1} K_{1}^{2}, r_{1} \gamma_{2} K_{2}^{2}, r_{1} r_{2}\left(1+K_{1}+K_{2}\right)\right)^{T}$ and $W=(0,0,1)^{T}$.

So, $\Omega_{1}=W^{T} \cdot F_{d}\left(E_{3}, d_{[T C]}\right) \quad=-\left.y\right|_{E_{3}}=0$,

$\Omega_{2}=W^{T}\left[D F_{d}\left(E_{3}, d_{[T C]}\right) V\right]=-v_{3} \neq 0$

and $\Omega_{3}=W^{T}\left[D^{2} F\left(E_{3}, d_{[T C]}\right)(V, V)\right]$

$$
\begin{aligned}
& =2 v_{3}\left[\left\{\frac{c_{1} \gamma_{1}\left\{2 K_{1}\left(1+K_{2}\right)+K_{1}^{2}\right\}}{\left(1+K_{1}+K_{2}\right)^{2}}-\frac{c_{2} \gamma_{2} K_{2}^{2}}{\left(1+K_{1}+K_{2}\right)^{2}}\right\} v_{1}\right. \\
& \left.+\left\{\frac{c_{2} \gamma_{2}\left\{2 K_{2}\left(1+K_{1}\right)+K_{2}^{2}\right\}}{\left(1+K_{1}+K_{2}\right)^{2}}-\frac{c_{1} \gamma_{1} K_{1}^{2}}{\left(1+K_{1}+K_{2}\right)^{2}}\right\} v_{2}\right] \neq 0 .
\end{aligned}
$$

By Sotomayor's Theorem, system (2.2) undergoes a transcritical bifurcation around $E_{3}$ at $d=d_{[T C]}$.

Theorem 8.2. System (2.2) undergoes a saddle-node bifurcation with respect to the bifurcation parameter $d$ around $E^{\star}\left(x_{1}^{\star}, x_{2}^{\star}, y^{\star}\right)$ when $G\left(x_{1}\right)=0$ (mentioned in (5.1)) has a double root in $\Omega$.

Proof. Let $x_{1[s n]}$ be a double root of $G\left(x_{1}\right)=0$ such that $0<x_{1[s n]}<K_{1}$. So, $G\left(x_{1[s n]}\right)=0=G^{\prime}\left(x_{1[s n]}\right)$ and $G^{\prime \prime}\left(x_{1[s n]}\right) \neq 0$. Let $r_{1[s n]}$ be the threshold value of $r_{1}$ such that for $r_{1}=r_{1[s n]}, x_{1[s n]}$ is a double root of $G\left(x_{1}\right)=0$. The non-trivial nullclines touch each other at $\left(x_{1[s n]}, r_{1[s n]}\right) \equiv E_{[s n]}^{\star}$. Let, $f_{1}\left(x_{1}, x_{2}, y\right)=x_{1} g_{1}\left(x_{1}, x_{2}, y\right), f_{2}\left(x_{1}, x_{2}, y\right)=x_{2} g_{2}\left(x_{1}, x_{2}, y\right)$ and $f_{3}\left(x_{1}, x_{2}, y\right)=y g_{3}\left(x_{1}, x_{2}, y\right) . \frac{d y^{\left(g_{1}\right)}}{d x_{1}}, \frac{d y^{\left(g_{1}\right)}}{d x_{2}}$ denote the slope of $g_{1}\left(x_{1}, x_{2}, y\right)=0 ; \frac{d y^{\left(g_{2}\right)}}{d x_{1}}, \frac{d y^{\left(g_{2}\right)}}{d x_{2}}$ denote the slope of $g_{2}\left(x_{1}, x_{2}, y\right)=0$ and $\frac{d y^{\left(g_{3}\right)}}{d x_{1}}, \frac{d y^{\left(g_{3}\right)}}{d x_{2}}$ denote the slope of $g_{3}\left(x_{1}, x_{2}, y\right)=0$.

Also, $\left.\frac{d y^{\left(g_{3}\right)}}{d x_{1}}\right|_{E_{[s n]}^{*}}=\left.\frac{d y^{\left(g_{2}\right)}}{d x_{1}}\right|_{E_{[s n]}^{*}}=\left.\frac{d y^{\left(g_{1}\right)}}{d x_{1}}\right|_{E_{[s n]}^{*}}$ and $\left.\frac{d y^{\left(g_{3}\right)}}{d x_{2}}\right|_{E_{[s n]}^{\star}}=\left.\frac{d y^{\left(g_{2}\right)}}{d x_{2}}\right|_{E_{[s n]}^{*}}=\left.\frac{d y^{\left(g_{1}\right)}}{d x_{2}}\right|_{E_{[s n]}^{*}}$.

Now, $\left.\frac{d y^{\left(g_{3}\right)}}{d x_{1}}\right|_{E_{[s n]}^{\star}}=-\left.\frac{\partial g_{3} / \partial x_{1}}{\partial g_{3} / \partial y}\right|_{E_{[s n]}^{\star}},\left.\frac{d y^{\left(g_{2}\right)}}{d x_{1}}\right|_{E_{[s n]}^{\star}}=-\left.\frac{\partial g_{2} / \partial x_{1}}{\partial g_{2} / \partial y}\right|_{E_{[s n]}^{\star}},\left.\frac{d y^{\left(g_{1}\right)}}{d x_{1}}\right|_{E_{[s n]}^{\star}}=-\left.\frac{\partial g_{1} / \partial x_{1}}{\partial g_{1} / \partial y}\right|_{E_{[s n]}^{\star}}$

and $\left.\frac{d y^{\left(g_{3}\right)}}{d x_{2}}\right|_{E_{[s n]}^{*}}=-\left.\frac{\partial g_{3} / \partial x_{2}}{\partial g_{3} / \partial y}\right|_{E_{[s n]}^{*}},\left.\frac{d y^{\left(g_{2}\right)}}{d x_{2}}\right|_{E_{[s n]}^{\star}}=-\left.\frac{\partial g_{2} / \partial x_{2}}{\partial g_{2} / \partial y}\right|_{E_{[s n]}^{\star}},\left.\frac{d y^{\left(g_{1}\right)}}{d x_{2}}\right|_{E_{[s n]}^{*}}=-\left.\frac{\partial g_{1} / \partial x_{2}}{\partial g_{1} / \partial y}\right|_{E_{[s n]}^{*}}$.

$\left.J\right|_{E^{*}}=\left(\begin{array}{lll}\frac{\partial f_{1}}{\partial x_{1}} & \frac{\partial f_{1}}{\partial x_{2}} & \frac{\partial f_{1}}{\partial y} \\ \frac{\partial f_{2}}{\partial x_{1}} & \frac{\partial f_{2}}{\partial x_{2}} & \frac{\partial f_{2}}{\partial y} \\ \frac{\partial f_{3}}{\partial x_{1}} & \frac{\partial f_{3}}{\partial x_{2}} & \frac{\partial f_{3}}{\partial y}\end{array}\right)=\left(\begin{array}{ccc}x_{1} \frac{d g_{1}}{d x_{1}} & x_{1} \frac{d g_{1}}{d x_{2}} & x_{1} \frac{d g_{1}}{d y} \\ x_{2} \frac{d g_{2}}{d x_{1}} & x_{2} \frac{d g_{2}}{d x_{2}} & x_{2} \frac{d g_{2}}{d y} \\ y \frac{d g_{3}}{d x_{1}} & y \frac{d g_{3}}{d x_{2}} & 0\end{array}\right)=\left(\begin{array}{ccc}a_{11} & a_{12} & a_{13} \\ a_{21} & a_{22} & a_{23} \\ a_{31} & a_{32} & 0\end{array}\right)$,

where, $a_{11}=-\left[\frac{r_{1} x_{1}^{\star}}{K_{1}}+\frac{\gamma_{1} x_{1}^{\star} y\left(1+x_{2}^{\star}\right)}{\left(1+x_{1}^{\star}+x_{2}^{\star}\right)^{2}}\right] ; a_{12}=\frac{\gamma_{1} x_{1}^{\star 2} y}{\left(1+x_{1}^{\star}+x_{2}^{\star}\right)^{2}} ; a_{13}=-\frac{\gamma_{1} x_{1}^{\star 2}}{1+x_{1}^{\star}+x_{2}^{\star}}$;

$a_{21}=\frac{\gamma_{2} x_{2}^{\star 2} y}{\left(1+x_{1}^{\star}+x_{2}^{\star}\right)^{2}} ; a_{22}=-\left[\frac{r_{2} x_{2}^{\star}}{K_{2}}+\frac{\gamma_{2} x_{2}^{\star} y^{\star}\left(1+x_{1}^{\star}\right)}{\left(1+x_{1}^{\star}+x_{2}^{\star}\right)^{2}}\right] ; a_{23}=-\frac{\gamma_{2} x_{2}^{\star 2}}{1+x_{1}^{\star}+x_{2}^{\star}} ;$ 
$a_{31}=\frac{c_{1} \gamma_{1} y^{\star}\left\{2 x_{1}^{\star}\left(1+x_{2}^{\star}\right)+x_{1}^{\star 2}\right\}}{\left(1+x_{1}^{\star}+x_{2}^{\star}\right)^{2}}-\frac{c_{2} \gamma_{2} x_{2}^{\star 2} y^{\star}}{\left(1+x_{1}^{\star}+x_{2}^{\star}\right)^{2}} ; a_{32}=\frac{c_{2} \gamma_{2} y^{\star}\left\{2 x_{2}^{\star}\left(1+x_{1}^{\star}\right)+x_{2}^{\star 2}\right\}}{\left(1+x_{1}^{\star}+x_{2}^{\star}\right)^{2}}-\frac{c_{1} \gamma_{1} x_{1}^{\star 2} y^{\star}}{\left(1+x_{1}^{\star}+x_{2}^{\star}\right)^{2}}$.

So, $\operatorname{Det}\left(\left.J\right|_{E_{[s n]}^{\star}}\right)=x_{1} x_{2} y\left[\frac{d g_{3}}{d x_{2}}\left(\frac{d g_{1}}{d y} \frac{d g_{2}}{d x_{1}}-\frac{d g_{1}}{d x_{1}} \frac{d g_{2}}{d y}\right)+\frac{d g_{3}}{d x_{1}}\left(\frac{d g_{1}}{d x_{2}} \frac{d g_{2}}{d y}-\frac{d g_{1}}{d y} \frac{d g_{2}}{d x_{2}}\right)\right]=0$ Thus the Jacobian matrix $\left.J\right|_{E_{[s n]}^{\star}}$ has a simple zero eigenvalue.

Here, $V=\left(-a_{13} a_{32}, a_{13} a_{31}, a_{11} a_{32}-a_{12} a_{31}\right)^{T}$ and $W=\left(-a_{23} a_{31}, a_{13} a_{31}, a_{11} a_{23}-a_{13} a_{21}\right)^{T}$.

So, $\Omega_{1}=W^{T} \cdot f_{r 1}\left(E^{\star}, r_{1[s n]}\right) \quad=-\left.\left(a_{11} a_{23}-a_{13} a_{21}\right) y^{\star}\right|_{E_{[s n]}^{\star}} \neq 0$

and $\Omega_{2}=W^{T}\left[D^{2} f\left(E^{\star}, r_{1[s n]}\right)(V, V)\right] \neq 0$.

Thus, from Sotomayor's Theorem, system (2.2) undergoes a non-degenerate saddle-node bifurcation around $E^{\star}$ at $r_{1}=r_{1[s n]}$.

Hopf Bifurcation at $E^{\star}\left(x_{1}^{\star}, x_{2}^{\star}, y^{\star}\right)$

Here, let us consider $\gamma_{1}$ as bifurcation parameter to check the instability of the equilibrium point $E^{\star}$. The characteristic equation of system (2.2) at $E^{\star}\left(x_{1}^{\star}, x_{2}^{\star}, y^{\star}\right)$ is

$\lambda^{3}+P_{1}\left(\gamma_{1}\right) \lambda^{2}+P_{2}\left(\gamma_{1}\right) \lambda+P_{3}\left(\gamma_{1}\right)=0$, where

where $P_{1}=-\left(a_{11}+a_{22}\right), P_{2}=a_{11} a_{22}-a_{12} a_{21}-a_{23} a_{32}-a_{13} a_{31}$ and $P_{3}=\left(a_{11} a_{23}-a_{13} a_{21}\right) a_{32}+a_{31}\left(a_{13} a_{22}-\right.$ $\left.a_{12} a_{23}\right)$

Here, $a_{11}=-\left[\frac{r_{1} x_{1}^{\star}}{K_{1}}+\frac{\gamma_{1} x_{1}^{\star} y\left(1+x_{2}^{\star}\right)}{\left(1+x_{1}^{\star}+x_{2}^{\star}\right)^{2}}\right] ; a_{12}=\frac{\gamma_{1} x_{1}^{\star 2} y}{\left(1+x_{1}^{\star}+x_{2}^{\star}\right)^{2}} ; a_{13}=-\frac{\gamma_{1} x_{1}^{\star 2}}{1+x_{1}^{\star}+x_{2}^{\star}} ;$

$a_{21}=\frac{\gamma_{2} x_{2}^{\star 2} y}{\left(1+x_{1}^{\star}+x_{2}^{\star}\right)^{2}} ; a_{22}=-\left[\frac{r_{2} x_{2}^{\star}}{K_{2}}+\frac{\gamma_{2} x_{2}^{\star} y^{\star}\left(1+x_{1}^{\star}\right)}{\left(1+x_{1}^{\star}+x_{2}^{\star}\right)^{2}}\right] ; a_{23}=-\frac{\gamma_{2} x_{2}^{\star 2}}{1+x_{1}^{\star}+x_{2}^{\star}} ;$

$a_{31}=\frac{c_{1} \gamma_{1} y^{\star}\left\{2 x_{1}^{\star}\left(1+x_{2}^{\star}\right)+x_{1}^{\star 2}\right\}}{\left(1+x_{1}^{\star}+x_{2}^{\star}\right)^{2}}-\frac{c_{2} \gamma_{2} x_{2}^{\star 2} y^{\star}}{\left(1+x_{1}^{\star}+x_{2}^{\star}\right)^{2}} ; a_{32}=\frac{c_{2} \gamma_{2} y^{\star}\left\{2 x_{2}^{\star}\left(1+x_{1}^{\star}\right)+x_{2}^{\star 2}\right\}}{\left(1+x_{1}^{\star}+x_{2}^{\star}\right)^{2}}-\frac{c_{1} \gamma_{1} x_{1}^{\star 2} y^{\star}}{\left(1+x_{1}^{\star}+x_{2}^{\star}\right)^{2}}$.

Theorem 8.3. If $E^{\star}$ exists with the feasibility conditions, then a simple Hopf bifurcation occurs at unique $\gamma_{1}=$ $\gamma_{1[H]}$, where $\gamma_{1[H]}$ is the unique positive root of the equation: $P_{1}\left(\gamma_{1}\right) P_{2}\left(\gamma_{1}\right)-P_{3}\left(\gamma_{1}\right)=0$ with $P_{1}\left(\gamma_{1[H]}\right), P_{2}\left(\gamma_{1[H]}\right)>$ 0 .

Proof. For $\gamma_{1}=\gamma_{1[H]}$, the characteristic equation of system (2.2) at $E^{\star}$ is

$\left(\lambda^{2}+P_{2}\right)\left(\lambda+P_{1}\right)=0$

which gives roots: $\lambda_{1}=i \sqrt{P_{2}}, \lambda_{2}=-i \sqrt{P_{2}}$ and $\lambda_{3}=-P_{1}$. So there exists a pair of purely imaginary eigenvalues and a strictly negative real eigenvalue. Also, $P_{i}\left(\gamma_{1}\right)$ are continuous functions of $\gamma_{1}$.

So, for $\gamma_{1}$ in a neighbourhood of $\gamma_{1[H]}$, the roots have the form:

$\lambda_{1}=k\left(\gamma_{1}\right)+i l\left(\gamma_{1}\right), \lambda_{2}=k\left(\gamma_{1}\right)-i l\left(\gamma_{1}\right), \lambda_{3}=-m\left(\gamma_{1}\right) ; k\left(\gamma_{1}\right), l\left(\gamma_{1}\right), m\left(\gamma_{1}\right)$ are real.

Next to check the transversality condition: $\left.\frac{d}{d \gamma_{1}}\left[\operatorname{Re}\left(\lambda_{j}\left(\gamma_{1}\right)\right)\right]\right|_{\gamma_{1}=\gamma_{1[H]}} \neq 0$, for $j=1,2$.

Put $\lambda\left(\gamma_{1}\right)=k\left(\gamma_{1}\right)+i l\left(\gamma_{1}\right)$ in $(8.1)$, we get

$(k+i l)^{3}+P_{1}(k+i l)^{2}+P_{2}(k+i l)+P_{3}=0$.

Taking derivative with respect to $\gamma_{1}$, we get

$3(k+i l)^{2}(\dot{k}+i \dot{l})+2 P_{1}(k+i l)(\dot{k}+i \dot{l})+P_{2}(\dot{k}+i \dot{l})+\dot{P}_{1}(k+i l)^{2}+\dot{P_{2}}(k+i l)+\dot{P_{3}}=0$

Comparing real and imaginary parts:

$M_{1} \dot{k}-M_{2} \dot{l}+M_{3}=0$,

$M_{2} \dot{k}+M_{1} \dot{l}+M_{4}=0$, 
where $M_{1}=3\left(k^{2}-l^{2}\right)+2 P_{1} k+P_{2} ; \quad M_{2}=6 k l+2 P_{1} l$;

$M_{3}=\dot{P_{1}}\left(k^{2}-l^{2}\right)+\dot{P_{2}} k+\dot{P_{3}} ; \quad M_{4}=2 \dot{P_{1}} k l+\dot{P_{2}} l$.

From the equations in (8.3):

$\dot{k}=-\frac{M_{2} M_{4}+M_{1} M_{3}}{M_{1}^{2}+M_{2}^{2}}$.

Now, $M_{3}=\dot{P_{1}}\left(k^{2}-l^{2}\right)+\dot{P_{2}} k+\dot{P_{3}} \neq \dot{P_{1}}\left(k^{2}-l^{2}\right)+\dot{P_{2}} k+\dot{P_{1}} P_{2}+\dot{P_{2}} P_{1}$.

At $\gamma_{1}=\gamma_{1[H]}$ :

Case-(1): $k=0 ; l=\sqrt{P_{2}}$.

So, $M_{1}=-2 P_{2} ; M_{2}=2 P_{1} \sqrt{P_{2}} ; M_{3} \neq P_{1} \dot{P_{2}} ; M_{4}=\dot{P_{2}} \sqrt{P_{2}}$

and $M_{2} M_{4}+M_{1} M_{3} \neq 2 P_{1} P_{2} \dot{P_{2}}-2 P_{1} P_{2} \dot{P_{2}}=0$.

Case-(2): $k=0 ; l=-\sqrt{P_{2}}$.

So, $M_{1}=-2 P_{2} ; M_{2}=-2 P_{1} \sqrt{P_{2}} ; M_{3} \neq P_{1} \dot{P_{2}} ; M_{4}=-\dot{P_{2}} \sqrt{P_{2}}$

and $M_{2} M_{4}+M_{1} M_{3} \neq 2 P_{1} P_{2} \dot{P_{2}}-2 P_{1} P_{2} \dot{P_{2}}=0$.

$$
\left.\therefore \frac{d}{d \gamma_{1}}\left[\operatorname{Re}\left(\lambda_{i}\left(\gamma_{1}\right)\right)\right]\right|_{\gamma_{1}=\gamma_{1[H]}}=-\frac{M_{2} M_{4}+M_{1} M_{3}}{M_{1}^{2}+M_{2}^{2}} \neq 0 .
$$

Also, $\lambda_{3}=-m=-P_{1}\left(\gamma_{1[H]}\right)<0$. Hence the theorem.

A co-dimensional 2 bifurcation is a cusp bifurcation where the critical equilibrium point has a zero eigenvalue and the quadratic coefficient for the saddle-node bifurcation vanishes. Two branches of saddle-node bifurcation curve or a saddle-node bifurcation curve and one transcritical bifurcation curve meet tangentially at the cusp bifurcation point forming a semi-cubic parabola. Moreover, in a two parametric plane a local bifurcation of co-dimension two occurs when Hopf-bifurcation curve and saddle-node curve intersect each other and this bifurcation is called Bogdanov-Takens bifurcation.

Theorem 8.4. The interior equilibrium point $E^{\star}$ of system (2.2) undergoes cusp bifurcation at the threshold $\left(r_{1}, \gamma_{1}\right)=\left(r_{1[C P]}, \gamma_{1[C P]}\right)$ around $E^{\star}\left(x_{1}^{\star}, x_{2}^{\star}, y^{\star}\right)$ when $G\left(x_{1}\right)=0$ in equation (5.1) has a triple root in $\Omega$.

Proof. Let $x_{1[C P]}$ be a triple root of $G\left(x_{1}\right)=0$, i.e., $G\left(x_{1[C P]}\right)=G^{\prime}\left(x_{1[C P]}\right)=G^{\prime \prime}\left(x_{1[C P]}\right)=0$ and $G^{\prime \prime \prime}\left(x_{1[C P]}\right) \neq 0$. Now proceeding as Theorem 8.2, it can be shown that the Jacobian matrix at $E_{[C P]}^{\star}\left(x_{1[C P]}, x_{2[C P]}, y_{[C P]}\right)$ (denoted by, $\left.\left.J\right|_{E_{[C P]}^{\star}}\right)$ has one zero eigenvalue. Moreover, the quadratic normal form coefficient for the saddle-node bifurcation at $E_{[C P]}^{\star}$ is given by $W^{T}\left[D^{2} f\left(E_{[C P]}^{\star}, r_{1[C P]}\right)(V, V)\right]=0$. Hence system (2.2) undergoes a co-dimensional two bifurcation which is known as cusp bifurcation at $\left(r_{1}, \gamma_{1}\right) \equiv$ $\left(r_{1[C P]}, \gamma_{1[C P]}\right)$ and the point $\left(r_{1[C P]}, \gamma_{1[C P]}\right)$ in the $r_{1}-\gamma_{1}$ parametric plane is the intersection of the two branches of saddle-node bifurcation, where they meet each other tangentially.

Theorem 8.5. The interior equilibrium point $E^{\star}$ of system (2.2) undergoes Bogdanov-Takens bifurcation at the threshold $\left(r_{1}, \gamma_{1}\right)=\left(r_{1[B T]}, \gamma_{1[B T]}\right)$ if $\left.\operatorname{Det}\left(\left.J\right|_{E^{*}}\right)\right|_{\left(r_{1[B T]}, \gamma_{1[B T]}\right)}=0$ and $\operatorname{Tr}\left(\left.\left.J\right|_{E^{*}}\right|_{\left(r_{1[B T]}, \gamma_{1[B T]}\right)}=0\right.$.

\section{Global Stability}

Theorem 9.1. If $c_{1} \gamma_{1} K_{1}^{2}+c_{2} \gamma_{2} K_{2}^{2}<d$, then $E_{3}\left(K_{1}, K_{2}, 0\right)$ is globally asymptotically stable.

Proof. From the first equation of system (2.2), we have

$$
\begin{aligned}
& \quad \frac{d x_{1}}{d t}=r_{1} x_{1}\left(1-\frac{x_{1}}{K_{1}}\right)-\frac{\gamma_{1} x_{1}^{2} y}{1+x_{1}+x_{2}} \leq r_{1} x_{1}\left(1-\frac{x_{1}}{K_{1}}\right) \\
& \Rightarrow \lim _{t \rightarrow \infty} x_{1}(t) \leq K_{1}
\end{aligned}
$$


From the second equation of system (2.2), we have

$$
\begin{aligned}
& \quad \frac{d x_{2}}{d t}=r_{2} x_{2}\left(1-\frac{x_{2}}{K_{2}}\right)-\frac{\gamma_{2} x_{2}^{2} y}{1+x_{1}+x_{2}} \leq r_{2} x_{2}\left(1-\frac{x_{2}}{K_{2}}\right) \\
& \Rightarrow \lim _{t \rightarrow \infty} x_{2}(t) \leq K_{2}
\end{aligned}
$$

For large time $t$ :

$$
\begin{aligned}
\frac{d y}{d t} & =\frac{c_{1} \gamma_{1} x_{1}^{2} y}{1+x_{1}+x_{2}}+\frac{c_{2} \gamma_{2} x_{2}^{2} y}{1+x_{1}+x_{2}}-d y \\
& \leq\left(c_{1} \gamma_{1} K_{1}^{2}+c_{2} \gamma_{2} K_{2}^{2}-d\right) y=-\mu y, \\
\Rightarrow & \lim _{t \rightarrow \infty} y(t)=0, \text { provided } c_{1} \gamma_{1} K_{1}^{2}+c_{2} \gamma_{2} K_{2}^{2}<d .
\end{aligned}
$$

Therefore, in the limit $x_{1}(t)$ and $x_{2}(t)$ are given by solutions of the equations: $\frac{d x_{1}}{d t}=r_{1} x_{1}\left(1-\frac{x_{1}}{K_{1}}\right)$ and $\frac{d x_{2}}{d t}=r_{2} x_{2}\left(1-\frac{x_{2}}{K_{2}}\right)$. Hence proved.

Remark: In system (2.2): global asymptotic stability of $E_{3}\left(K_{1}, K_{2}, 0\right)$ guarantees that it is locally asymptotically stable.

Theorem 9.2. The interior equilibrium point $E^{\star}$ is globally asymptotically stable in the region:

$\Psi_{2}=\left\{\left(x_{1}, x_{2}, y\right) \in \mathbb{R}_{+}^{3}: x_{1}>\frac{2 r_{1} K_{1}\left(1+K_{1}+K_{2}\right)}{r_{1}\left(1+K_{1}+K_{2}\right)+K_{1} \gamma_{1} y^{\star}}, x_{2}>\frac{2 r_{2} K_{2}\left(1+K_{1}+K_{2}\right)}{r_{2}\left(1+K_{1}+K_{2}\right)+K_{2} \gamma_{2} y^{\star}}\right\}$

if $c_{1} \gamma_{1} x_{1}^{\star}+c_{2} \gamma_{2} x_{2}^{\star}<d$ and $c_{1} r_{1} x_{1}^{\star}+c_{2} r_{2} x_{2}^{\star}>d y^{\star}$ hold.

Proof. Consider an appropriate Lyapunov function:

$$
V\left(x_{1}, x_{2}, y\right)=c_{1}\left[x_{1}-x_{1}^{\star}-x_{1}^{\star} \log \left(\frac{x_{1}}{x_{1}^{\star}}\right)\right]+c_{2}\left[x_{2}-x_{2}^{\star}-x_{2}^{\star} \log \left(\frac{x_{2}}{x_{2}^{\star}}\right)\right]+\left[y-y^{\star}-y^{\star} \log \left(\frac{y}{y^{\star}}\right)\right] .
$$

Here $V\left(x_{1}, x_{2}, y\right)$ is a positive definite function for all $\left(x_{1}, x_{2}, y\right)$ except at $\left(x_{1}^{\star}, x_{2}^{\star}, y^{\star}\right)$. The time derivative of $V$ computed along the solutions of system (2.2) is given by

$$
\begin{aligned}
\dot{V} & =c_{1}\left(1-\frac{x_{1}^{\star}}{x_{1}}\right)\left[r_{1} x_{1}\left(1-\frac{x_{1}}{K_{1}}\right)-\frac{\gamma_{1} x_{1}^{2} y}{1+x_{1}+x_{2}}\right]+c_{2}\left(1-\frac{x_{2}^{\star}}{x_{2}}\right)\left[r_{2} x_{2}\left(1-\frac{x_{2}}{K_{2}}\right)\right. \\
& \left.-\frac{\gamma_{2} x_{2}^{2} y}{1+x_{1}+x_{2}}\right]+\left(1-\frac{y^{\star}}{y}\right)\left[\frac{c_{1} \gamma_{1} x_{1}^{2} y}{1+x_{1}+x_{2}}+\frac{c_{2} \gamma_{2} x_{2}^{2} y}{1+x_{1}+x_{2}}-d y\right] \\
& =c_{1} r_{1} x_{1}\left(1-\frac{x_{1}}{K_{1}}\right)+c_{2} r_{2} x_{2}\left(1-\frac{x_{2}}{K_{2}}\right)-c_{1} r_{1} x_{1}^{\star}\left(1-\frac{x_{1}}{K_{1}}\right)-c_{2} r_{2} x_{2}^{\star}\left(1-\frac{x_{2}}{K_{2}}\right) \\
& +\frac{c_{1} \gamma_{1} x_{1}^{\star} x_{1} y}{1+x_{1}+x_{2}}+\frac{c_{2} \gamma_{2} x_{2}^{\star} x_{2} y}{1+x_{1}+x_{2}}-\frac{c_{1} \gamma_{1} x_{1}^{2} y^{\star}}{1+x_{1}+x_{2}}-\frac{c_{2} \gamma_{2} x_{2}^{2} y^{\star}}{1+x_{1}+x_{2}}-d y+d y^{\star} \\
& \leq c_{1} r_{1} x_{1}-\frac{c_{1} r_{1}}{K_{1}} x_{1}^{2}+c_{2} r_{2} x_{2}-\frac{c_{2} r_{2}}{K_{2}} x_{2}^{2}-c_{1} r_{1} x_{1}^{\star}+\frac{c_{1} r_{1} x_{1}^{\star}}{K_{1}} x_{1}-c_{2} r_{2} x_{2}^{\star}+\frac{c_{2} r_{2} x_{2}^{\star}}{K_{2}} x_{2} \\
& +c_{1} \gamma_{1} x_{1}^{\star} y+c_{2} \gamma_{2} x_{2}^{\star} y-\frac{c_{1} \gamma_{1} x_{1}^{2} y^{\star}}{1+K_{1}+K_{2}}-\frac{c_{2} \gamma_{2} x_{2}^{2} y^{\star}}{1+K_{1}+K_{2}}-d y+d y^{\star} \\
& \leq 2 c_{1} r_{1} x_{1}+2 c_{2} r_{2} x_{2}-\frac{c_{1} r_{1}}{K_{1}} x_{1}^{2}-\frac{c_{2} r_{2}}{K_{2}} x_{2}^{2}-c_{1} r_{1} x_{1}^{\star}-c_{2} r_{2} x_{2}^{\star} \\
& +y\left(c_{1} \gamma_{1} x_{1}^{\star}+c_{2} \gamma_{2} x_{2}^{\star}-d\right)-\frac{c_{1} \gamma_{1} x_{1}^{2} y^{\star}}{1+K_{1}+K_{2}}-\frac{c_{2} \gamma_{2} x_{2}^{2} y^{\star}}{1+K_{1}+K_{2}}+d y^{\star} \\
& =c_{1} x_{1}\left[2 r_{1}-x_{1}\left(\frac{r_{1}}{K_{1}}+\frac{\gamma_{1} y^{\star}}{1+K_{1}+K_{2}}\right)\right]+c_{2} x_{2}\left[2 r_{2}-x_{2}\left(\frac{r_{2}}{K_{2}}+\frac{\gamma_{2} y^{\star}}{1+K_{1}+K_{2}}\right)\right] \\
& +y\left(c_{1} \gamma_{1} x_{1}^{\star}+c_{2} \gamma_{2} x_{2}^{\star}-d\right)-\left(c_{1} r_{1} x_{1}^{\star}+c_{2} r_{2} x_{2}^{\star}-d y^{\star}\right)
\end{aligned}
$$




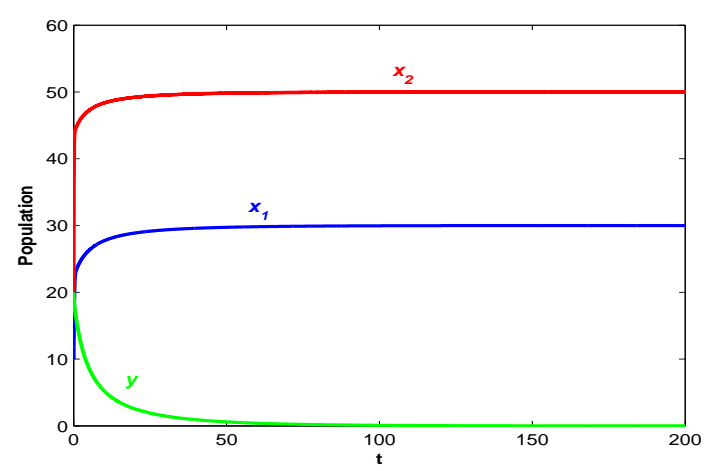

(2.a)

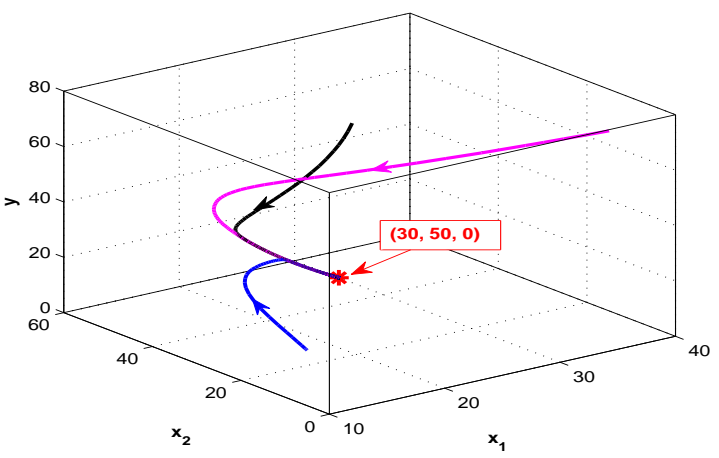

(2.b)

Figure 2: Stable behaviour of $E_{3}$ for $d=0.85$. Parametric values have been taken from Table 1.

So, $\dot{V}<0$ when the stated conditions are satisfied in $\Psi_{2}$. Also $\dot{V}=0$ when $\left(x_{1}, x_{2}, y\right)=\left(x_{1}^{\star}, x_{2}^{\star}, y^{\star}\right)$. Hence $\dot{V}$ is negative definite under some parametric restrictions and LaSalle theorem [10] implies global asymptotic stability of $E^{\star}$.

\section{Numerical Simulation}

Numerical simulation is a vital part for any model system as this support the analytical results. In this work, we have considered a two-prey and one-predator system where the predator population switches between two prey population according to their abundance. We have considered five sets of parameters to analyze the local dynamics of the system. To draw Figure 2 and Figure 3, we set parametric values in Table-1. Figure 2 shows that the trajectory approaches (for $d=0.85)$ to the planer equilibrium point $E_{3}(30,50,0)$ where two prey species coexist in a steady state. But if the predator mortality rate starts to decrease, then below a certain value $\left(d_{[T C]}\right)$ the trajectory approaches towards interior equilibrium point $E^{\star}$ instead of $E_{3}$. Figure 3 shows that the trajectory approaches (for $d=0.3)$ to $E^{\star}(8.656,24.661,70.519)$ where all the species coexist in a steady state. So, from the steady state of $E_{3}$ if $d$ goes below a threshold value, then $E_{3}$ switches from stable to unstable state and the system undergoes a transcritical bifurcation around $E_{3}$ at $d=d_{[T C]}=0.81$ (see Figure 4).

Next we consider another set of values of the parameters as mentioned in Table 2 to draw Figure 5Figure 10. Figure 5 shows that the system undergoes consequent two Hopf bifurcations taking $\gamma_{1}$ as bifurcation parameter. Here $H_{1}$ and $H_{2}$ denote the Hopf bifurcation points where $H_{1}:\left(x_{1}^{\star}, x_{2}^{\star}, y^{\star} ; \gamma_{1[H 1]}\right) \equiv$ $(13.38,38.30,136.87 ; 0.280037)$ and $H_{2}:\left(x_{1}^{\star}, x_{2}^{\star}, y^{\star} ; \gamma_{1[H 2]}\right) \equiv(7.41,53.53,114.94 ; 0.716046)$. At $H_{1}$ and $H_{2}$, the first Lyapunov coefficients $\left(l_{1}\right)$ are $-5.339167 e^{-05}$ and $-4.377044^{-05}$ respectively which implies both the Hopf bifurcations are supercritical. And hence we get two limit point cycles (in Figure (5.b)) denoted by $L P C_{1}$ and $L P C_{2}$ respectively at $H_{1}$ and $H_{2}$ where $L P C_{1}:\left(\right.$ period $=1.613107 e^{0}$, parameter $\left.=\gamma_{1[H 1]}\right)$ with normal form coefficient $=-1.178702 e^{+00}$ and $L P C_{2}:\left(\right.$ period $=1.514331 e^{0}$, parameter $\left.=\gamma_{1[H 2]}\right)$ with normal form coefficient $=1.332611 e^{-01}$. Figure (6.a) and (7.a) shows that for $\gamma_{1}=0.15$, the system approaches to interior steady state $E^{\star}(19.45,30.24,166.99)$. After crossing $\gamma_{1[H 1]}=0.280037$, figure (6.b) and (7.b) depict an oscillating behaviour of the system and occurrence of a stable limit cycle around unstable equilibrium

Table 1: Parameter values used for numerical simulation of system (2.2)

\begin{tabular}{l|l|l|l|l|l|l|l}
\hline \multicolumn{2}{c}{ Parametric Values: Set-1 } \\
\hline$r_{1}$ & $r_{2}$ & $K_{1}$ & $K_{2}$ & $\gamma_{1}$ & $\gamma_{2}$ & $c_{1}$ & $c_{2}$ \\
\hline 10 & 20 & 30 & 50 & 0.4 & 0.2 & 0.1 & 0.06 \\
\hline
\end{tabular}




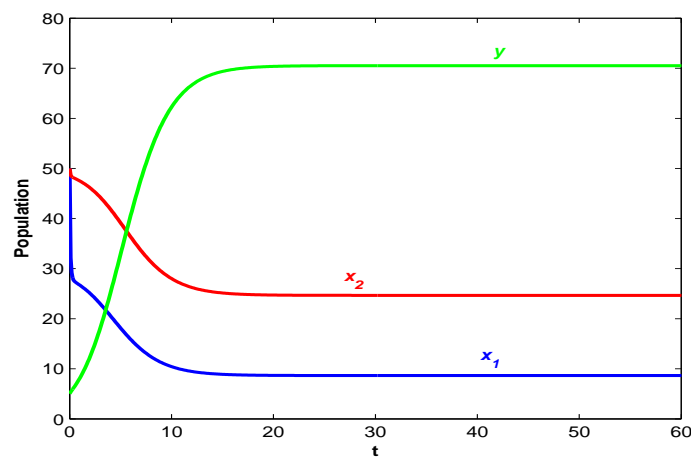

(3.a)

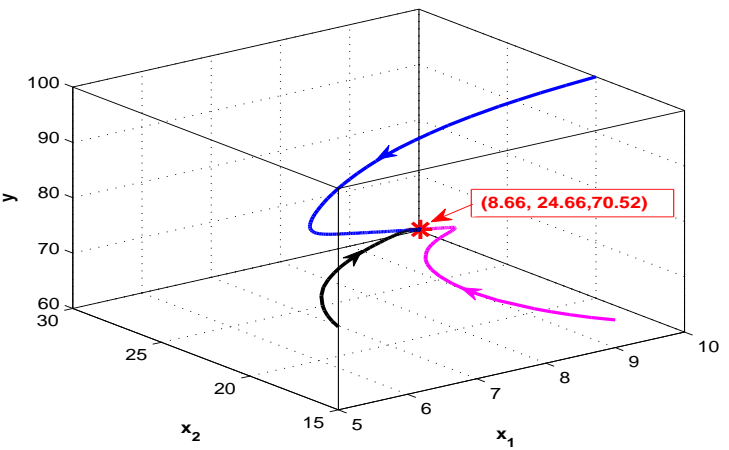

(3.b)

Figure 3: Stable behaviour of $E^{\star}$ for $d=0.3$. Parametric values have been taken from Table 1.

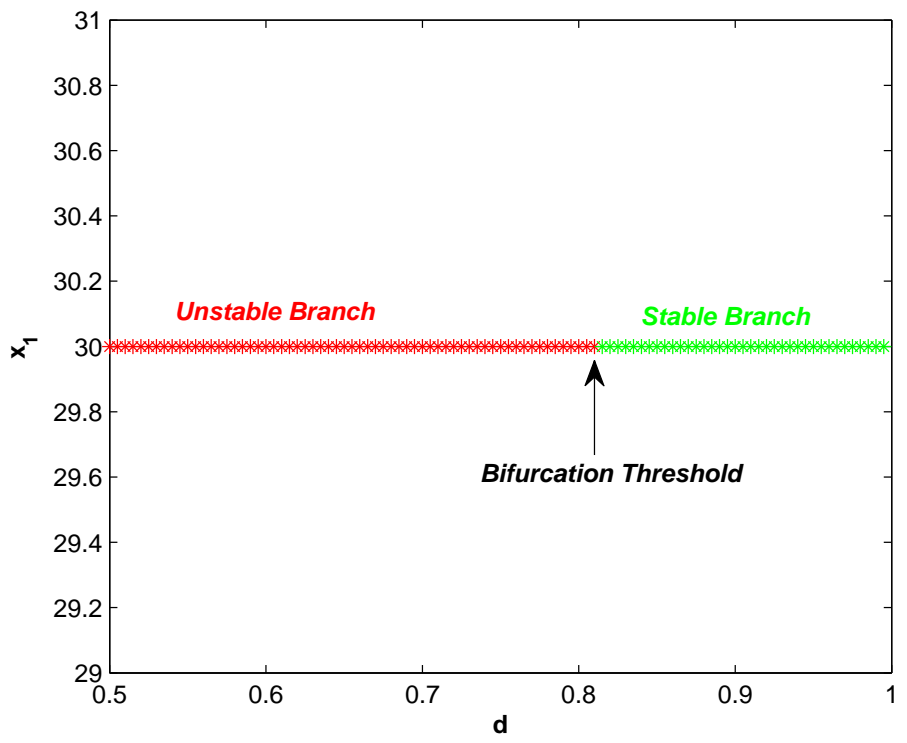

Figure 4: Transcritical bifurcation around $E_{3}$ taking $d$ as bifurcation parameter. Parametric values have been taken from Table 1.

Table 2: Parameter values used for numerical simulation of system (2.2)

\begin{tabular}{l|l|l|l|l|l|l|l|l}
\hline \multicolumn{1}{c}{ Parametric Values: Set-2 } \\
\hline$r_{1}$ & $r_{2}$ & $K_{1}$ & $K_{2}$ & $\gamma_{1}$ & $\gamma_{2}$ & $c_{1}$ & $c_{2}$ & $d$ \\
\hline 10 & 20 & 500 & 8000 & 0.5 & 0.2 & 0.7 & 0.06 & 1 \\
\hline
\end{tabular}



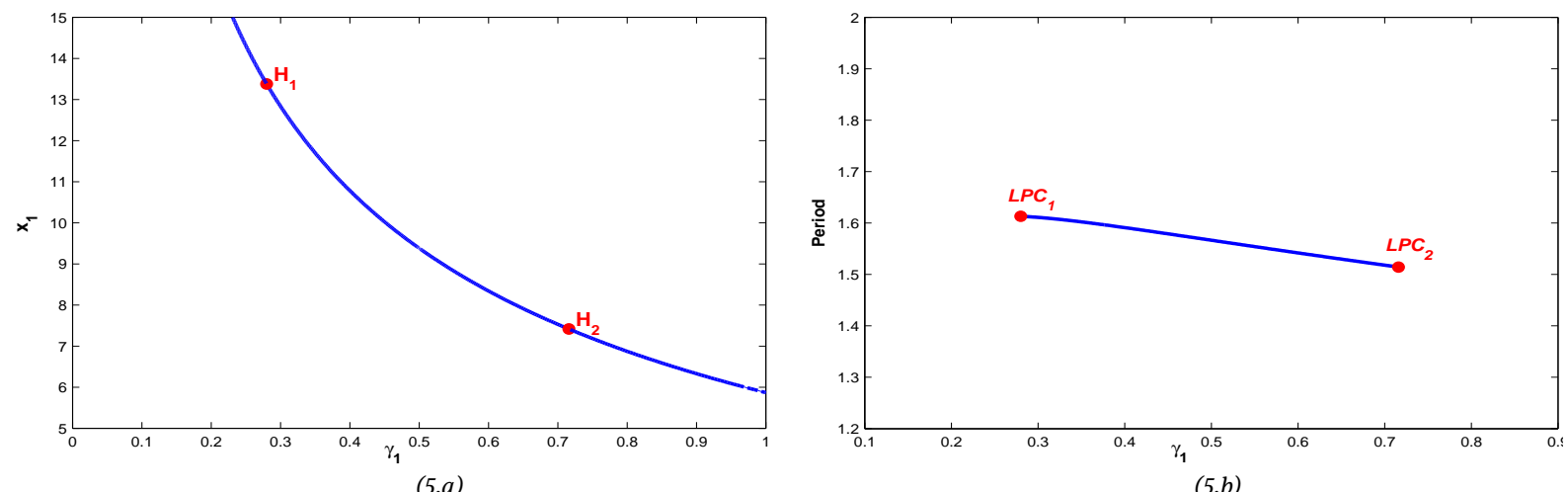

Figure 5: Occurrence of two Hopf bifurcations taking $\gamma_{1}$ as bifurcation parameter. Parametric values have been taken from Table 2.

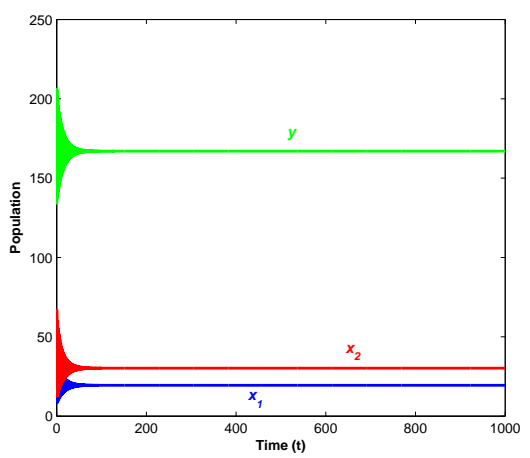

(6.a)

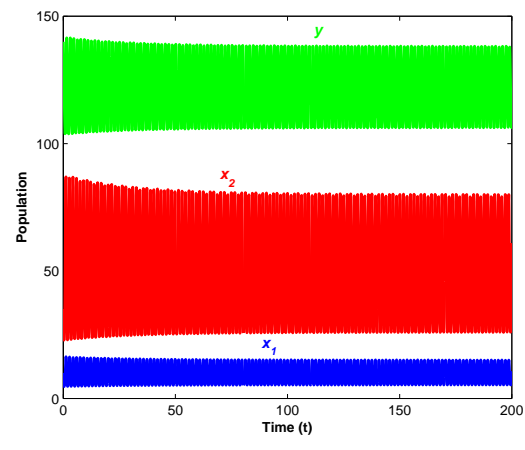

(6.b)

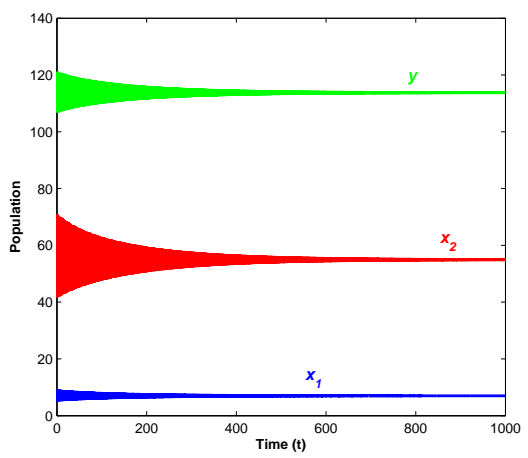

(6.c)

Figure 6: Time-series plot of $E^{\star}$ for different values of $\gamma_{1}$ : (6.a) Stable behaviour for $\gamma_{1}=0.15<\gamma_{1[H 1]}$, (6.b) Oscillating behaviour for $\gamma_{1}=0.5 \in\left(\gamma_{1[H 1]}, \gamma_{1[H 2]}\right)$ and (6.c) Stable behaviour for $\gamma_{1}=0.78>\gamma_{1[H 2]}$. Parametric values have been taken from Table 2.

point $E^{\star}\left(9.381,47.52,121.123\right.$ ) (as $l_{1}<0$ ) for $\gamma_{1}=0.5$. After crossing $\gamma_{1[H 2]}=0.716046$ the limit cycle disappears and figure (6.c) and (7.c) show that for $\gamma_{1}=0.78>\gamma_{1[\mathrm{H} 2]}$, the system again converges to stable interior equilibrium point $E^{\star}(6.99,54.95,113.76)$. Hence, system $(2.2)$ undergoes two hopf bifurcations at $\gamma_{1}=\gamma_{1\left[H_{1}\right]}$ and $\gamma_{1}=\gamma_{1\left[H_{2}\right]}$ respectively. Figure 8 exhibits the hopf bifurcation diagram taking $\gamma_{1}$ as the bifurcation parameter and we can conclude that the consumption rate of predator for the first prey population can stabilize as well as destabilize the system.

From figure (6.b) and (7.b), it is shown that for $\gamma_{1}=0.5$ and $d=1$, the system performs oscillation and a stable limit cycle occurs around the unstable equilibrium point $(9.381,47.52,121.123)$. But decreasing value of $d$ can settle the system into stable state through Hopf bifurcation. Figure 9 shows that for $d=0.1$, the system tends to stable interior equilibrium point $E^{\star}(1.067,5.343,138.596)$. Figure 10 portraits the Hopf bifurcation diagram taking $d$ as the bifurcation parameter and so, the system undergoes a supercritical Hopf bifurcation at $d=d_{[H]}=0.952601\left(l_{1}=-5.543133 e^{-05}\right)$ with the equilibrium components $(8.945,45.281,121.272)$.

To draw the one-dimensional saddle-node bifurcation and two-dimensional BT and cusp bifurcations, we have considered another set of parametric values mentioned in Table 3. From the coefficients of equation (5.1), it is observed that the system has either one or three equilibrium points depending on the choice of parametric values. Figure 11 shows that the system undergoes two non-degenerate saddle-node bifurcations taking $r_{1}$ as the bifurcating parameter which are denoted by $L P_{1}$ and $L P_{2}$ where $L P_{1}:\left(E^{1 *} ; r_{1\left[L P_{1}\right]}\right) \equiv$ $(1.42,9.63,0.00092 ; 0.000055683744)$ with normal form coefficient $a=-2.020627 e^{-05}$ and $L P_{2}$ : 


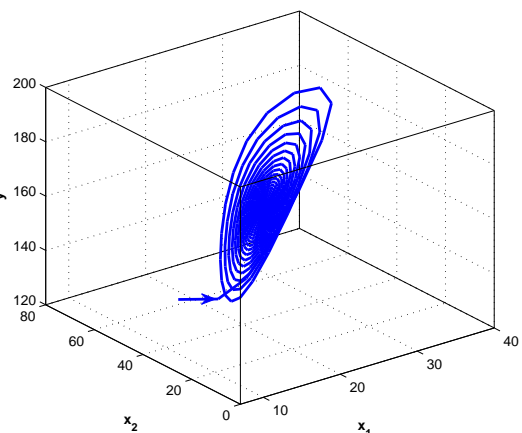

(7.a)

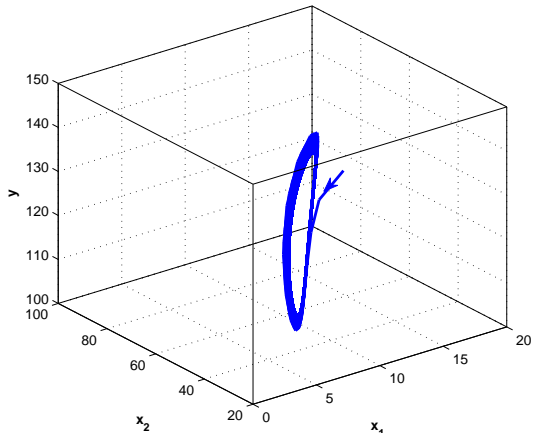

(7.b)

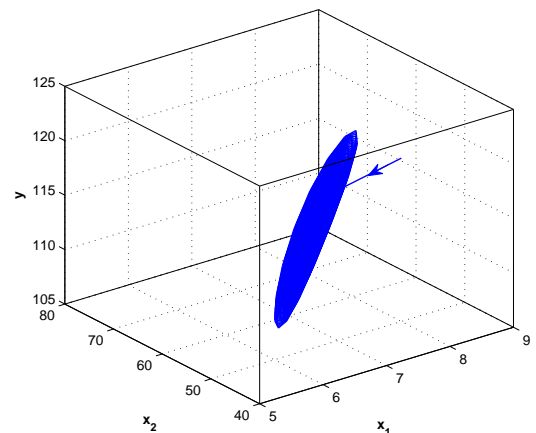

(7.c)

Figure 7: Change of behaviour of $E^{\star}$ for different values of $\gamma_{1}$ : (7.a) Stable behaviour for $\gamma_{1}=0.15<\gamma_{1[H 1]}$, (7.b) Occurrence of limit cycle for $\gamma_{1}=0.5 \in\left(\gamma_{1[H 1]}, \gamma_{1[H 2]}\right)$ and (7.c) limit cycle disappears for $\gamma_{1}=0.78>\gamma_{1[H 2]}$. Parametric values have been taken from Table 2.

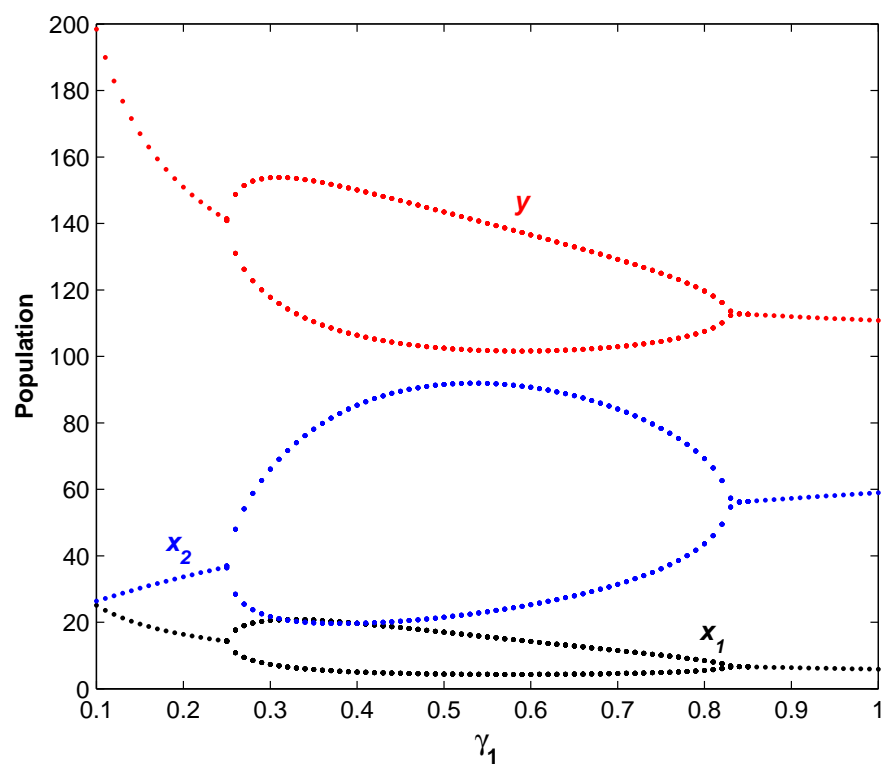

Figure 8: Hopf Bifurcation around $E^{\star}$ taking $\gamma_{1}$ as bifurcation parameter. Parametric values have been taken from Table 2 .

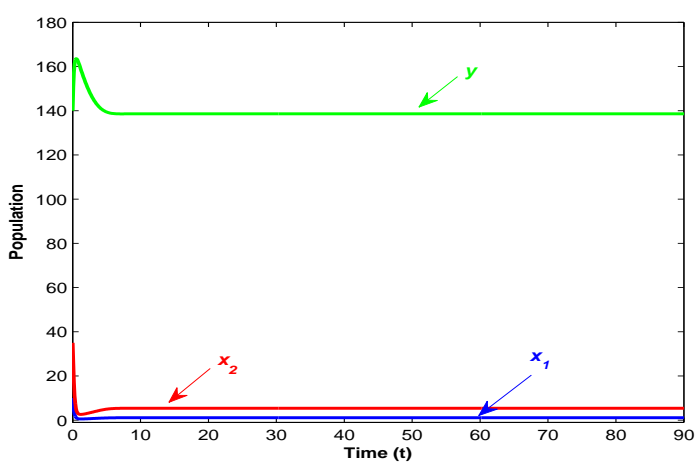

(9.a)

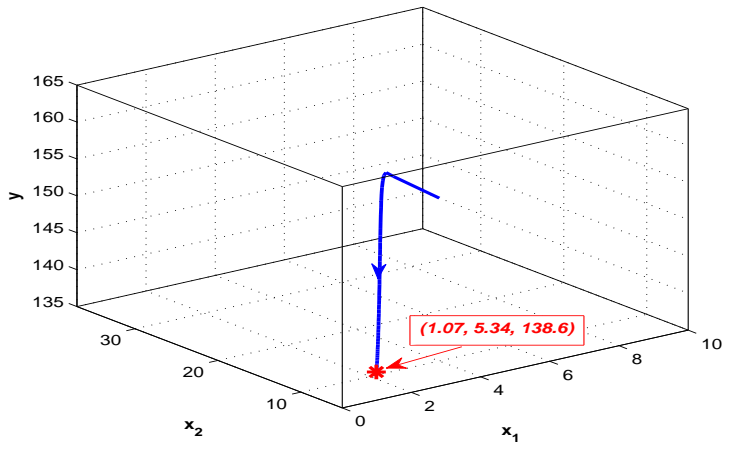

(9.b)

Figure 9: Stable behaviour of $E^{\star}$ for $d=0.1<d_{[H]}$. Parametric values have been taken from Table 2 . 


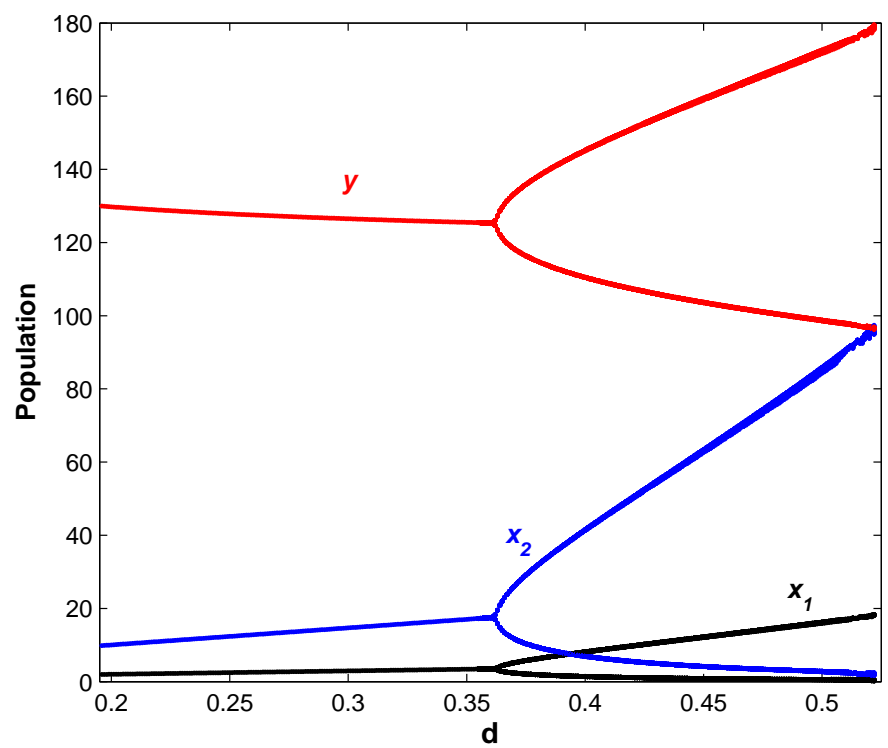

Figure 10: Hopf Bifurcation around $E^{\star}$ taking $d$ as bifurcation parameter. Parametric values have been taken from Table 2 .

Table 3: Parameter values used for numerical simulation of system (2.2)

Parametric Values: Set-3

\begin{tabular}{l|l|l|l|l|l|l|l|l}
\hline$r_{1}$ & $r_{2}$ & $K_{1}$ & $K_{2}$ & $\gamma_{1}$ & $\gamma_{2}$ & $c_{1}$ & $c_{2}$ & $d$ \\
\hline 0.1 & 0.01 & 50 & 10 & 0.5 & 0.5 & 0.9 & 0.5 & 2 \\
\hline
\end{tabular}

$\left(E^{2^{\star}} ; r_{1\left[L P_{2}\right]}\right) \equiv(1.48,9.65,0.00089 ; 0.000055681786)$ with normal form coefficient $a=-2.064553 e^{-05}$. From the calculations and also from Figure 11, it is observed that when $r_{1}$ lies below $r_{1\left[L P_{2}\right]}$ and above $r_{1\left[L P_{1}\right]}$, there exists only one stable interior equilibrium point but when $r_{1}$ lies between $\left(r_{1\left[L P_{2}\right]}, r_{1\left[L P_{1}\right]}\right)$, then there are three equilibria out of which two equilibria are stable and one is a saddle point. So, a case of bistability has occur in this case. Bistability is a phenomenon which indicates that the trajectories can converge to two different equilibrium points for same parametric values depending on the choice of initial points. Figure 12 shows that for $r_{1}=0.000055682$, a trajectory starting from $x_{0}=(1.4,9,0.0009)$ converges to $E^{1^{\star}}(1.39,9.62,0.00094)$ but a trajectory starting from $x_{0}=(1.5,10,0.0008)$ tends to $E^{2 \star}(1.49,9.65,0.00088)$. In fact, from the calculations, it is observed that for $r_{1}=0.000055681784<r_{1\left[L P_{2}\right]}$, there is only one stable equilibrium point $E^{\star}(1.38,9.62,0.00094)$; for $r_{1}=0.000055682 \in\left(r_{1\left[L P_{2}\right]}, r_{1\left[L P_{1}\right]}\right)$, there are two stable interior equilibrium points $E^{1^{\star}}(1.39,9.62,0.00094), E^{2^{\star}}(1.49,9.65,0.00088)$ and one saddle point $E^{3^{\star}}(1.46,9.64,0.00089)$ and for $r_{1}=0.00005568375>r_{1\left[L P_{1}\right]}$, there is again only one stable equilibrium point $E^{\star}(1.51,9.65,0.00087)$.

Figure 13 and Figure 14 show the occurrence of two-dimensional bifurcations along with one dimensional local bifurcations. Figure 13 shows that between $L P_{1}$ and $L P_{2}$, there is a point where the two saddle-node bifurcation curves meet tangentially forming a cusp bifurcation denoted by " $\mathrm{CP}$ ". Figures 13 and 14 depict that at $\left(r_{1[C P]}, \gamma_{1[C P]}\right) \equiv\left(5.5731201 e^{-05}, 0.50022185\right)$, system (2.2) undergoes a non-degenerate cusp bifurcation with equilibrium components $(1.45,9.64,0.0009)$ and the normal form coefficient $\left(c=-2.187865 e^{-05}\right)$. Moreover, "BT" bifurcation occurs when saddle-node curve and Hopf curve intersect. The system undergoes Bogdanov-Takens (BT) bifurcation at $\left(r_{1[B T]}, \gamma_{1[B T]}\right) \equiv\left(6.2935815 e^{-09}, 0.37038128\right)$ with the equilibrium components $\left(2.999866,9.999971,8.2004 e^{-8}\right)$ and the normal form coefficients 

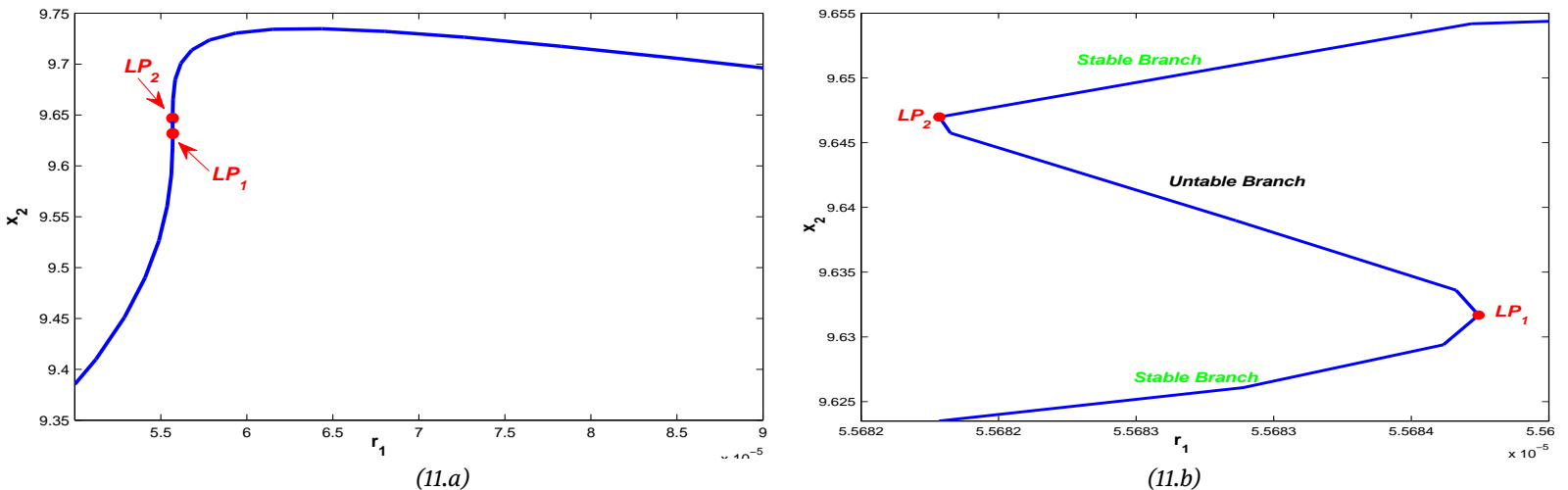

Figure 11: Occurrence of saddle-node bifurcations taking $r_{1}$ as bifurcation parameter. Parametric values have been taken from Table 3. Figure (11.b) is the enlarged version of Figure (11.a).

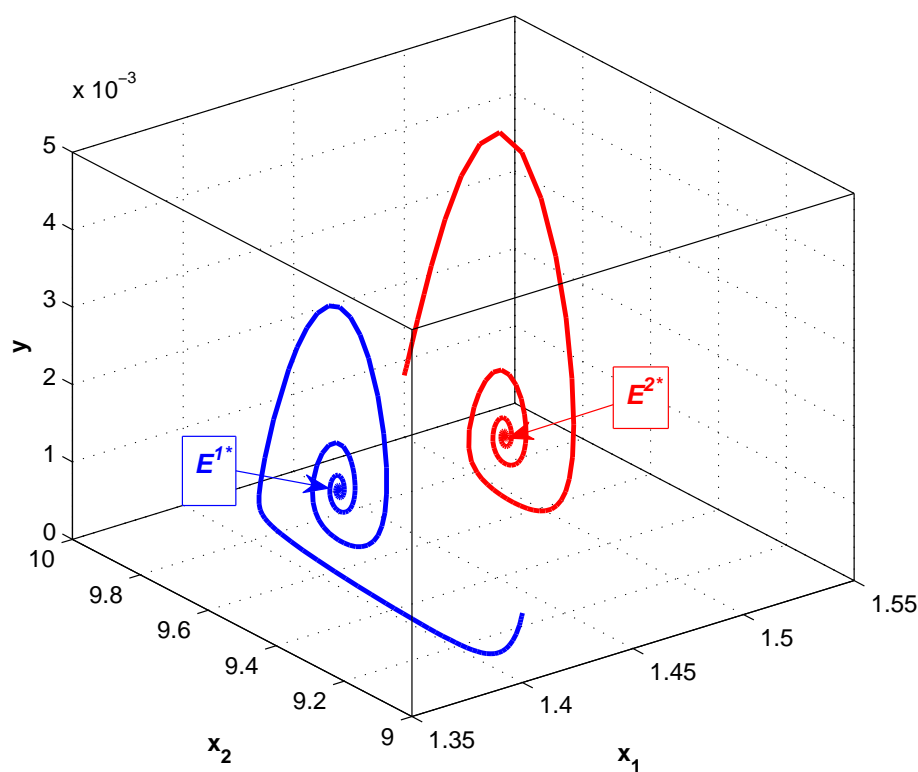

Figure 12: Bistability of the system for interior equilibrium point $E_{1^{\star}}$ and $E^{2 \star}$. Parametric values have been taken from Table 3. 


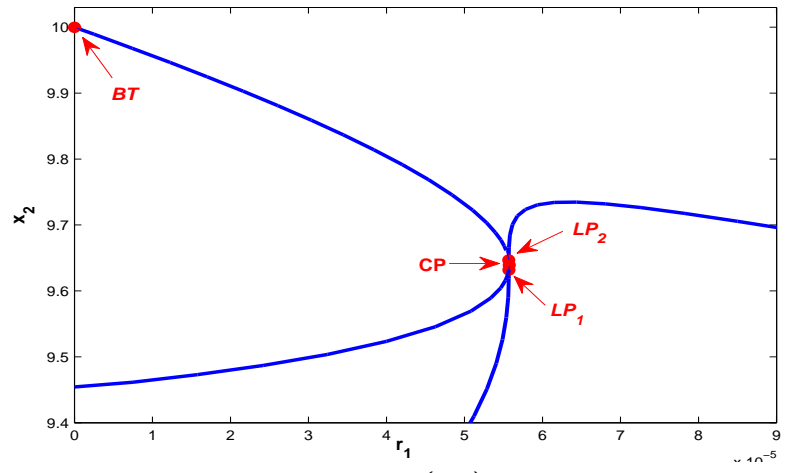

(13.a)

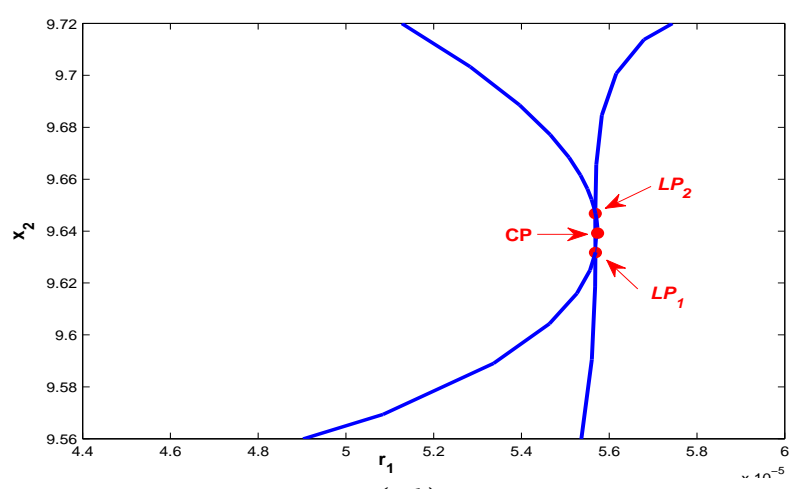

(13.b)

Figure 13: Occurrence of one-dimensional, i.e., saddle-node bifurcations $\left(L P_{1}, L P_{2}\right)$ and two dimensional, i.e., BT bifurcation and Cusp bifurcation. Parametric values have been taken from Table 3. Figure (13.b) is the enlarged version of Figure (13.a).

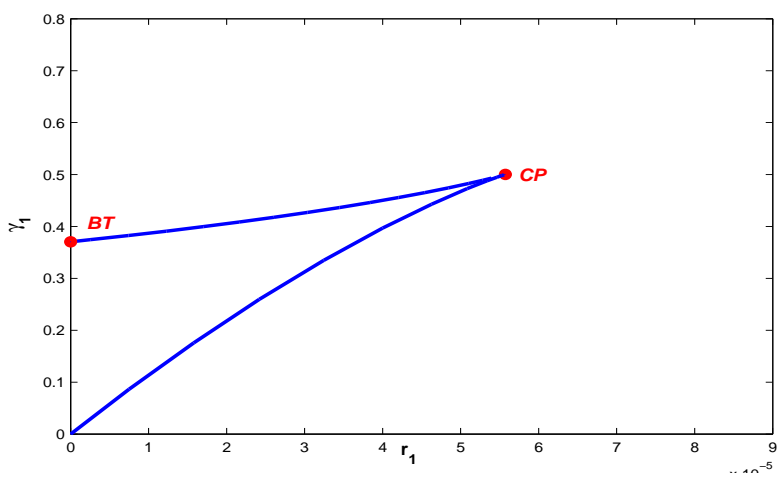

Figure 14: Two-dimensional bifurcation diagram in $r_{1}-\gamma_{1}$ plane where $B T:\left(r_{1[B T]}, \gamma_{1[B T]}\right)$ and $C P:\left(r_{1[C P]}, \gamma_{1[C P]}\right)$. Parametric values have been taken from Table 3.

$(a, b)=\left(-4.651499 e^{-10}, 6.424405 e^{-06}\right)$.

Another set of parametric values has been considered in Table 4 to analyse the count of predator population for increasing intrinsic growth rate of first prey population $\left(r_{1}\right)$ when the growth rate of second prey species $\left(r_{2}\right)$ is smaller than the first prey. Figure 15 shows that when the consumption rate of predator for first prey population $\left(\gamma_{1}\right)$ increases, the predator population increases for increasing $r_{1}$. In this case, if the predator consumes the first prey at a lesser quantity and chooses the second prey frequently, then the growth of predator becomes much smaller than the other cases. On the other hand, if the predator consumes the first prey at a higher rate, then their growth becomes higher enough due to the adequacy of first prey.

Further the parametric values mentioned in Table 5 are chosen to analyse the count of predator population for increasing intrinsic growth rate of second prey population $\left(r_{2}\right)$ when the growth rate of first prey species $\left(r_{1}\right)$ is smaller than the second prey. Figure 16 shows that when the consumption rate of predator for second prey population $\left(\gamma_{2}\right)$ increases, the predator population increases for increasing $r_{2}$. In this case, if

Table 4: Parameter values used for numerical simulation of system (2.2)

Parametric Values: Set-4

\begin{tabular}{l|l|l|l|l|l|l}
\hline$r_{2}$ & $K_{1}$ & $K_{2}$ & $\gamma_{2}$ & $c_{1}$ & $c_{2}$ & $d$ \\
\hline 0.01 & 10 & 50 & 0.5 & 0.5 & 0.5 & 2 \\
\hline
\end{tabular}




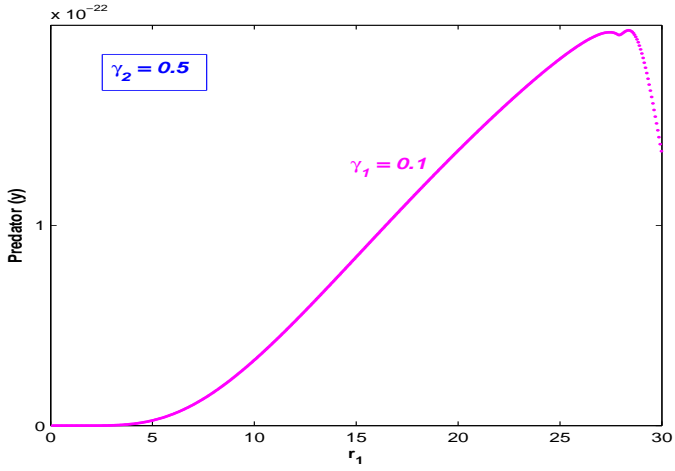

(15.a)

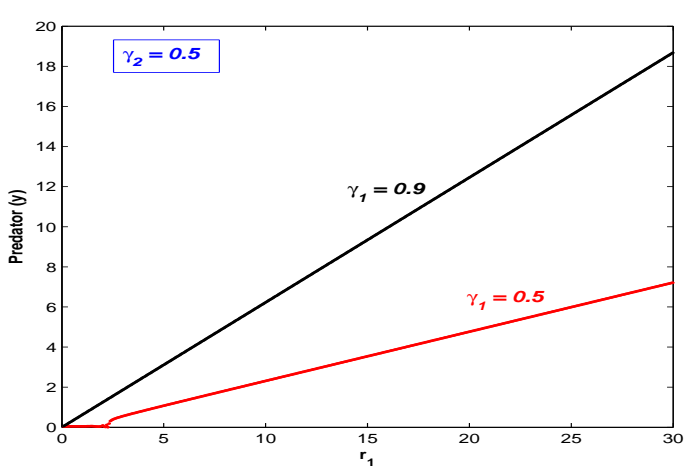

(15.b)

Figure 15: Variation of predator population $(y)$ for increasing value of $r_{1}$ with different values of $\gamma_{1}$. Parametric values have been taken from Table 4.

Table 5: Parameter values used for numerical simulation of system (2.2)

\begin{tabular}{l|l|l|l|l|l|l}
\hline \multicolumn{7}{c}{ Parametric Values: Set-5 } \\
\hline$r_{1}$ & $K_{1}$ & $K_{2}$ & $\gamma_{1}$ & $c_{1}$ & $c_{2}$ & $d$ \\
\hline 0.01 & 50 & 10 & 0.5 & 0.5 & 0.5 & 2 \\
\hline
\end{tabular}
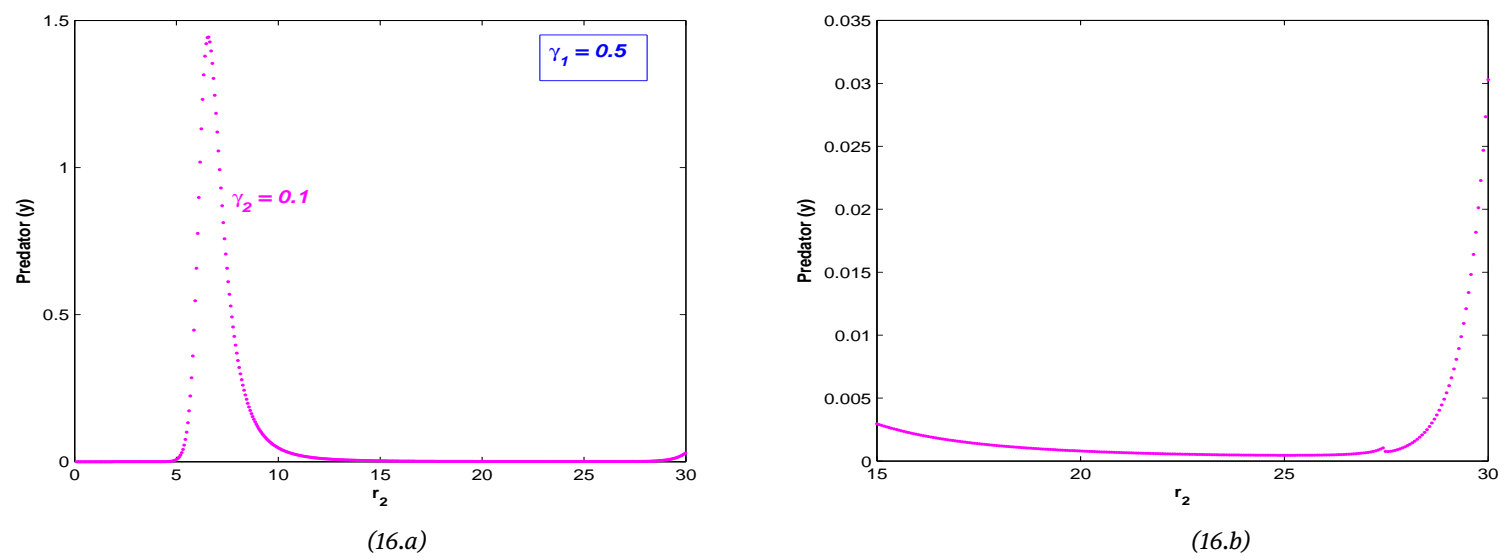

the predator consumes the second prey at a lesser quantity and chooses the first prey for hunting, then the growth of predator become smaller. On the other hand, because the system has a sufficient number of second prey, if the predator consumes more of the second prey, their growth becomes higher. So, adopting switching strategy based on adequacy of prey population increases the overall growth of the predator population.

\section{Discussion}

Switching of prey population is a kind of consumption strategy of predator which is frequency-dependent, i.e., the predator prefers to consume that prey which presents in the system with sufficient amount. Prey switching can be applied when the predator has a choice between two or more prey species. Oaten \& Murdoch have made an experiment on snails to observe their switching behaviour on prey species mussel and barnacle [19]. The snails prefer to hunt the prey with higher density. Taking Tubificids and Drosophila as the prey species, switching behaviour is observed in Guppies when one prey is available in lesser amount in environment [17]. For the case for stoneflies, Elliott has shown that the mature larvae of Isoperla grammatica and 


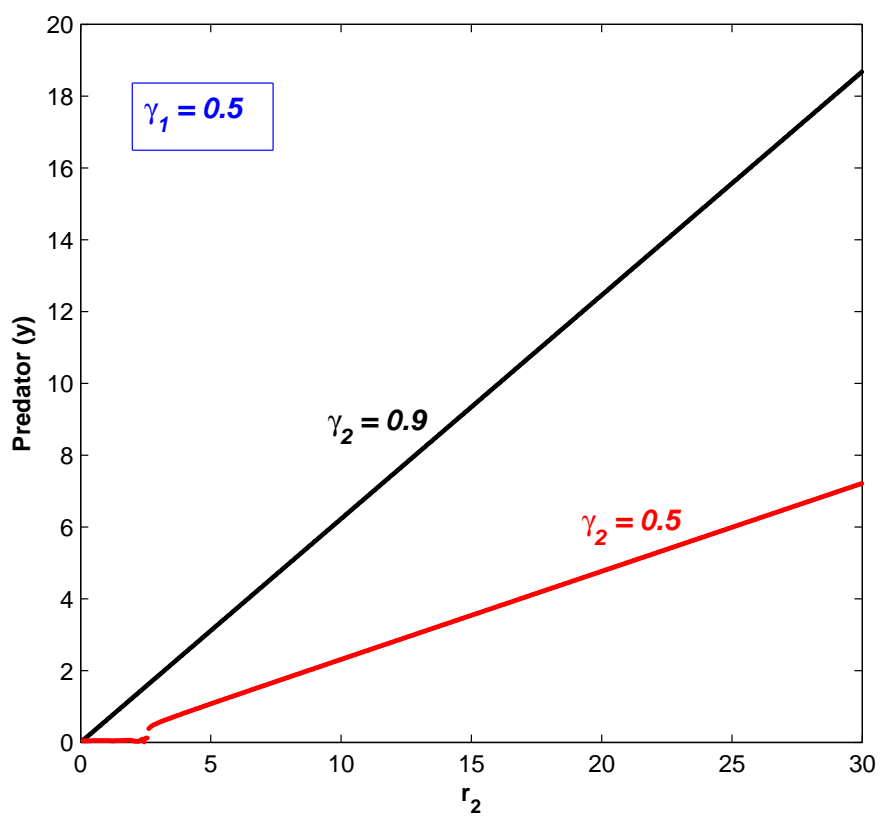

(16.c)

Figure 16: Variation of predator population ( $y$ ) for increasing value of $r_{2}$ with different values of $\gamma_{2}$. Parametric values have been taken from Table 5. Here figure (16.b) is zoomed figure of (16.a).

Perlodes microcephalus prefer to consume Baetis rhodani over Chironomus sp. when the abundance of Baetis is higher in the system [5]. We have formulated the underlying model to observe how this prey switching behavior makes an impact on the growth of the predator population.

In this work, two prey-one predator system has been considered where the predator population chooses the prey population for consumption according to their abundance. Both the prey population here grow with logistic law. The predator is considered here as a specialist one as the growth rate of predator depends only on the prey population $x_{1}$ and $x_{2}$. The proposed model is biologically well-defined as the system variables are positive and bounded for all time. The extinction criterion shows that if the overall growth rate of predator fails to exceed the mortality rate, then with time, the predator will go extinct from the system. Stability analysis shows that the planer equilibrium point containing both prey population and also the coexisting equilibrium points are asymptotically stable under some parametric restrictions. The mortality rate of predator (d) plays a crucial role to control the system dynamics. The predator-free equilibrium changes from unstable to a stable position through transcritical bifurcation when $d$ crosses the bifurcation threshold. Further, the consumption rate of predator for first prey species $\left(\gamma_{1}\right)$ also plays an important role. It is observed in the numerical simulation that, $\gamma_{1}$ has a stabilizing as well as destabilizing effect. It means an increasing value of $\gamma_{1}$ can turn a stable system into an oscillating system through Hopf bifurcation but for further increment, the system recovers its stability through another Hopf bifurcation and converges to steady-state. Instead of $\gamma_{1}$, regulating the predator mortality rate coefficient $(d)$ can lead to a system with oscillation. The system can exhibit two saddle-node bifurcations by regulating the intrinsic growth rate of first prey population $\left(r_{1}\right)$. Moreover, bistable behaviour is also observed in the system in a certain range of $r_{1}$. In the range $\left(r_{1\left[L P_{2}\right]}, r_{1\left[L P_{1}\right]}\right)$, there are three interior equilibrium points out of which two points are locally asymptotically stable and one is a saddle point. So, a trajectory tends to either of those stable equilibria depending on the choice of the initial point. One of the benefits of prey switching is that the growth rate of predator always increases as they can choose that particular prey which is abundant in the system. If the intrinsic growth rate of second prey population is lesser than the first prey, then Figure 15 reveals that when the consumption rate for first prey lies below the predatory rate of second prey, the predator population can not increase that much but when 
the consumption rate for first prey becomes higher than the consumption rate for the second prey, then the count of predator population becomes significantly higher. Also, when the intrinsic growth rate of the first prey population is lower than the second prey, Figure 16 depicts that the predator population increases with a higher rate for a higher predatory rate of the second prey. So, instead of searching the prey which is inadequate in the system, the predator should consume that prey that is present in ample amount as it benefits their growth. The overall analysis reveals that the switching effect helps to increase the predator population and as the switching depends on the adequacy of prey species, so, the prey species can save themselves from the verge of extinction.

The overall analysis shows that the proposed model exhibits interesting results but this model can be refined further. The consumption term here is dependent only on the prey population but one may consider another consumption term which is dependent on the predator population too. Moreover, incorporation of 'gestation delay' can also be considered as the consumption procedure is not instantaneous and the predator needs some time to digest the food before further hunting. Also, the incorporation of the Allee effect can make the work more realistic. The future model can be formulated considering all these facts to make it more realistic.

Acknowledgements: The authors are grateful to the anonymous referees and Dr. Shan Zhao, Journal Editor, for their careful reading, valuable comments and helpful suggestions, which have helped them to improve the presentation of this work significantly. The first author (Sangeeta Saha) is thankful to the University Grants Commission, India for providing SRF.

Author contribution: All the authors have accepted responsibility for the entire content of this submitted manuscript and approved submission.

Financial Support: This research received no specific grant from any funding agency, commercial or nonprofit sectors.

Conflict of Interests Statement: The authors have no conflicts of interest to disclose.

Ethics Statement: This research did not require ethical approval.

\section{References}

[1] Abrams, P., \& Matsuda, H. Consequences of behavioral dynamics for the population dynamics of predator-prey systems with switching. Population Ecology, 46(1), (2004) 13-25. doi:10.1007/s10144-003-0168-2

[2] Baum, W. M. On two types of deviation from the matching law: bias and undermatching. Journal of the experimental analysis of behavior, 22(1) (1974) 231-42. doi:10.1901/jeab.1974.22-231

[3] Bergelson, J. M. A Mechanistic Interpretation of Prey Selection by Anax junius Larvae (Odonata: Aeschnidae). Ecology, 66(6), (1985) 1699-1705. doi:10.2307/2937365

[4] Comins, H. N. \& Hassell, M. P. Predation in multi-prey communities. Journal of Theoretical Biology, 62(1), (1976) 93-114. doi:10.1016/0022-5193(76)90053-9

[5] Elliott, J. M. Prey switching in four species of carnivorous stoneflies. Freshwater Biology, 49(6), (2004) 709-720. doi:10.1111/j.1365-2427.2004.01222.x

[6] Freedman, H.I. \& Ruan, S. Uniform persistence in functional differential equations. J Differ Equ, 115, (1995) 173-192.

[7] Gentleman, W., Leising, A., Frost, B., Strom, S., \& Murray, J. Functional responses for zooplankton feeding on multiple resources: a review of assumptions and biological dynamics. Deep Sea Research Part II: Topical Studies in Oceanography, 50(22-26), (2003) 2847-2875. doi:10.1016/j.dsr2.2003.07.001

[8] Hale, J.K. Theory of functional Differential Equations, Springer-Verlag, Heidelberg, 1977.

[9] Hughes, R.N. \& Croy, M.I. An experimental analysis of frequency-dependent predation (Switching) in the 15-spines Stickleback, Spinachia spinachia. J. Anim. Ecol., 62, (1993) 341-352.

[10] La Salle, J. The stability of dynamical systems (SIAM), 1976.

[11] Matsuda, H., Kawasaki, K., Shigesada, N., Teramoto, E., \& Ricciardi, L. M. Switching effect on the stability of the preypredator system with three trophic levels. Journal of Theoretical Biology, 122(3), (1986) 251-262. doi:10.1016/s00225193(86)80118-7 
[12] Mondal, S. \& Samanta, G. P. Dynamics of an additional food provided predator-prey system with prey refuge dependent on both species and constant harvest in predator. Physica A: Statistical Mechanics and its Applications, 534:122301, 2019. https://doi.org/10.1016/j.physa.2019.122301

[13] Mondal, S. \& Samanta, G. P. Dynamics of a delayed predator-prey interaction incorporating nonlinear prey refuge under the influence of fear effect and additional food. Journal of Physics A: Mathematical and Theoretical 53, (2020) 295601. https://doi.org/10.1088/1751-8121/ab81d8

[14] Mondal, S. and Samanta, G. P. Impact of fear on a predator-prey system with prey-dependent search rate in deterministic and stochastic environment. Nonlinear Dynamics, 2021. https://doi.org/10.1007/s11071-021-06435-x

[15] Murdoch, W. W. Switching in General Predators: Experiments on Predator Specificity and Stability of Prey Populations. Ecological Monographs, 39(4), (1969) 335-354. doi:10.2307/1942352

[16] Murdoch, W. W. \& Oaten, A. Predation and population stability. Advances in Ecological Research 9, (1975) 1-131.

[17] Murdoch, W., Avery, S., \& Michael E. B. Smyth. Switching in Predatory Fish. Ecology, 56(5), (1975) $1094-1105$. doi:10.2307/1936149

[18] Murray, J. Mathematical Biology (ii): Spatial models and biomedical applications (3 ${ }^{\text {rd }}$ edition), 2003.

[19] Oaten, A., \& Murdoch, W. W. Switching, Functional Response, and Stability in Predator-Prey Systems. The American Naturalist, 109(967), (1975) 299-318. doi:10.1086/282999

[20] Perko, L. Differential Equations and Dynamical Systems. Springer-Verlag, New York, 2001.

[21] Real L.A. Predator Switching and the Interpretation of Animal Choice Behavior: The Case for Constrained Optimization. In: Hughes R.N. (eds) Behavioural Mechanisms of Food Selection. NATO ASI Series (Series G: Ecological Sciences), 20 (1990) 1-21, Springer, Berlin, Heidelberg.

[22] Saha, S., Maiti, A. \& Samanta, G.P. A Michaelis-Menten Predator-Prey Model with Strong Allee Effect and Disease in Prey Incorporating Prey Refuge. International Journal of Bifurcation and Chaos 28(6), (2018) 1850073 (21 pages). https://doi.org/10.1142/S0218127418500736

[23] Saha, S. \& Samanta, G.P. Analysis of a predator-prey model with herd behaviour and disease in prey incorporating prey refuge. International Journal of Biomathematics 12(01), (2019) 1950007. https://doi.org/10.1142/S1793524519500074.

[24] Saha, S. \& Samanta, G.P. Local dynamics of a predator-prey community in a moderate period of time. Energ. Ecol. Environ. 5, (2020) 47-60. https://doi.org/10.1007/s40974-019-00146-1

[25] Sahoo, D. and Samanta, G. P. Impact of fear effect in a two prey-one predator system with switching behaviour in predation. Differential Equations and Dynamical Systems, 2021. https://doi.org/10.1007/s12591-021-00575-7

[26] Siddon, C. E., \& Witman, J. D. Behavioral Indirect Interactions: Multiple Predator Effects And Prey Switching in The Rocky Subtidal. Ecology, 85(11), (2004) 2938-2945. doi:10.1890/03-0519

[27] Tansky, M. Switching effect in prey-predator system. Journal of Theoretical Biology, 70, (1978) 263-271.

[28] Teramoto, E., Kawasaki, K., \& Shigesada, N. Switching effect of predation on competitive prey species. Journal of Theoretical Biology, 79(3), (1978) 303-315. doi:10.1016/0022-5193(79)90348-5

[29] Tinbergen, L. The natural control of insects in pine woods. I. Factors influencing the intensity of predation by songbirds. Archives néerlandaises de zoologie, 13, (1960) 265-336,

[30] Van Baalen, M., Křivan, V., van Rijn, P. C. J. \& Sabelis, M. W. Alternative Food, Switching Predators, and the Persistence of Predator-Prey Systems. The American Naturalist, 157(5), (2001) 512-524. doi:10.1086/319933 